ISSN: $1130-2887$

DOI: http://dx.doi.org/10.14201/alh2013651544

\title{
CUANDO LOS PARTIDOS POLÍTICOS SE ENCUENTRAN CON SUS VOTANTES: UN ANÁLISIS DE LOS VÍNCULOS POLÍTICOS A TRAVÉS DE LAS REDES PARTIDARIAS Y LAS EXPECTATIVAS DISTRIBUTIVAS EN ARGENTINA Y CHILE When parties meet voters: assessing political linkages through partisan networks and distributive expectations in Argentina and Chile
}

\author{
Ernesto CALVO \\ University of Maryland, Estados Unidos \\ \ecalvo@uma.edu \\ María Victoria MuRILLO \\ Columbia University, Estados Unidos \\ $\triangle m m 2140 @ c o l u m b i a . e d u$
}

BIBLID [1130-2887 (2013) 65, 15-44]

Fecha de recepción: 1 de julio del 2013

Fecha de aceptación: 18 de octubre del 2013

RESUMEN: Este artículo propone un nuevo método comparado para el estudio de los vínculos entre los partidos políticos y los electores. Visto desde los electores, la principal distinción entre los partidos clientelares y los programáticos se encuentra en el modo de acceso a los beneficios públicos. En el primer caso, partidos clientelares, las redes partidarias median en el acceso a los beneficios públicos. En el segundo caso, partidos programáticos, los beneficiarios son definidos por el gobierno mediante política pública y el acceso a los mismos es independiente de las redes de distribución partidarias. Por medio de una novedosa metodología para medir redes partidarias, este artículo muestra que diferentes mecanismos de acceso a los beneficios moldean las expectativas distributivas de los votantes y la naturaleza de su vínculo con los partidos políticos. El método utiliza encuestas originales de Argentina y Chile y muestra la variación en los vínculos entre los partidos políticos y los votantes, midiendo la variación al interior de los países así como entre países.

Palabras clave: redes, partidos políticos, clientelismo, vínculos políticos. 
ERNESTO CALVO Y MARÍA VICTORIA MURILLO

CUANDO LOS PARTIDOS POLÍTICOS SE ENCUENTRAN CON SUS VOTANTES: UN ANÁLISIS DE LOS VÍNCULOS

POLÍTICOS A TRAVÉS DE LAS REDES PARTIDARIAS Y LAS EXPECTATIVAS DISTRIBUTIVAS EN ARGENTINA Y CHILE

ABSTRACT: This article provides a new comparative methodology for the study of partyvoter linkages from the perspective of voters where the critical question that distinguishes clientelistic from programmatic parties is access to publicly provided benefits. In the former case, partisan networks mediate access to goods. In the latter, beneficiaries are defined by policy and access is independent from partisan distribution networks. We show that these different access mechanisms shape voters' distributive expectations and the nature of their linkages to political parties by developing a unique methodology to measure party networks. We test it using original survey data from Argentina and Chile and show variation both across and within countries on party-voter linkages based on differential access to benefits and parties' organizational capacity.

Key words: networks, political parties, clientelism, political linkages.

\section{INTRODUCCIÓN ${ }^{1}$}

Este artículo proporciona un nuevo marco conceptual para el estudio comparado de los vínculos programáticos y no programáticos entre los partidos políticos y sus electores. Desde la perspectiva de los votantes, la pregunta principal que distingue los vínculos clientelares de los programáticos es «¿Cómo voy a recibir el beneficio que me ofrece un partido político?». En efecto, partidos clientelares y programáticos se distinguen por la forma en que los votantes son definidos como potenciales receptores de beneficios públicos. En el primer caso -relaciones clientelares- las redes partidarias definen cuáles votantes merecen o no merecen recibir beneficios, con múltiples redes políticas que arbitran el acceso a los mismos. En el segundo caso -relaciones programáticas-, el grupo de beneficiarios es definido por el gobierno y el acceso a los beneficios no depende de las redes de distribución partidarias. En este artículo se analiza la forma en que el tipo de acceso a los beneficios -mediado o no por las redes partidarias- moldea las expectativas distributivas de los votantes, describiendo efectos heterogéneos entre los distintos partidos políticos y entre distintos países. En particular, se mide el efecto que tienen los vínculos políticos (relaciones o conexiones ${ }^{2}$ entre miembros de los distintos partidos políticos y los votantes) sobre las expectativas distributivas de los electores. Con este objetivo, se propone una novedosa metodología para el análisis de las redes partidarias, la cual puede ser utilizada para el estudio de diversos fenómenos sociales en forma comparada.

La ola democratizadora que comenzó a mediados de la década de 1970 llevó a una revalorización conceptual de los vínculos entre los partidos políticos y los votantes. En la actualidad, se ha puesto en evidencia que los preconceptos sobre la estructura

1. La versión final y definitiva de este artículo ha sido publicada en inglés en Comparatives Political Studies, 2012, vol. 46 (7): 851-882. «SAGE Publications Ltd.», All rights reserved. (C) [as appropriate]. Esta traducción estuvo a cargo de Carola Lustig y fue revisada por los autores.

2. El análisis de redes sociales distingue nodos (votantes y partidos en el análisis) y relaciones (interacciones entre individuos). En este artículo se utilizan indistintamente los términos vínculo, conexión, o relación para describir el contacto personal entre individuos. El interés es el contacto personal entre votantes y distintas categorías de miembros partidarios (militantes, candidatos, adherentes). 
organizativa de los partidos y los vínculos programáticos con sus votantes, como fuera definido por la tradición sociológica de Europa Occidental, no explican de modo adecuado el comportamiento de los votantes y la dinámica de la competencia partidaria en la mayoría de las nuevas democracias (Keefer y Vlaicu 2008; Kitschelt 2000; Kitschelt y Wilkinson 2007; Magaloni, Díaz-Cayeros y Estévez 2007).

Para aportar un marco teórico que explique la naturaleza de la representación en las nuevas democracias, surgió una amplia literatura que contrasta las características organizacionales de partidos programáticos con las características de los partidos clientelares ${ }^{3}$. Este marco teórico presume que las élites de los partidos programáticos responden a las preferencias de aquellos votantes con quienes comparten una mayor afinidad ideológica y, consecuentemente, dictan políticas públicas redistributivas en beneficio de dichos electorados. Por su parte, los partidos clientelares se especializan en la distribución de bienes privados a un menú restringido de votantes. La mayor parte de la literatura emergente sobre políticas redistributivas en las nuevas democracias, por tanto, distingue entre partidos programáticos y partidos clientelares a partir de observar el tipo de beneficio -público o privado- que reparten a sus votantes.

Resulta problemático, sin embargo, distinguir los partidos programáticos de los clientelares por el tipo de beneficio que otorgan. Tal como observó Kitschelt (2000), es dif́́cil clasificar los objetivos programáticos o clientelares de un partido según el tipo de bienes públicos universales, colectivos o privados que reparte. Esta valoración subjetiva entorpece las comparaciones sobre los vínculos entre los partidos políticos y los votantes. El acceso a un seguro de desempleo, por ejemplo, puede estar mediado por referentes partidarios, militantes y punteros en un país y puede estar mediado por una agencia burocrática en otro. Los cargos públicos pueden ser asignados por concurso público o pueden ser otorgados discrecionalmente de acuerdo con las preferencias de autoridades políticas en la organización partidaria. La inscripción de beneficiarios en programas sociales puede depender de referencias personales y contactos directos con líderes partidarios o puede estar basada en reglas burocráticas que identifican un grupo social con necesidades específicas. En otras palabras, los mismos bienes públicos o privados pueden ser distribuidos de un modo programático o clientelar, garantizando distintos objetivos políticos.

En este artículo interesa entender el mecanismo mediante el cual se decide cuáles votantes son receptores de bienes públicos. Analizamos si los votantes perciben que las redes partidarias median o no en el acceso a los beneficios. Se entiende a las redes partidarias como estructuras sociales compuestas por individuos (nodos que describen a miembros partidarios y votantes) y vínculos personales (conexiones o relaciones que

3. Al comienzo de la década de 1960 y de 1970, surgió una extensa literatura sobre el clientelismo que enfatizó la noción de reciprocidad (E. C. BANFIELD y J. Q. Wilson 1963; S. W. SCHMidT 1977; J. C. Sсотт 1972). En contraste, investigaciones recientes tienen una perspectiva instrumental sobre la relación clientelar, entendiendo el intercambio clientelar como un contrato -difícil de hacer cumplirentre patrones y clientes (G. W. Cox y M. D. MCCuBbins 1986; A. Dixit y J. LONDREGAN 1996, 1998; H. KitscheLt y S. Wilkinson 2007; F. C. SChafFer 2007; S. C. STOKes 2007). 
describen contactos entre pares de individuos en una red política). A su vez, se considera que un mayor número de conexiones entre votantes y miembros de un partido refleja un mayor grado de proximidad a dicho partido. Con esta definición como punto de partida, este artículo se propone medir la estructura y el tamaño de las redes partidarias así como la importancia que los votantes asignan al tipo de mecanismo (redes políticas o proximidad ideológica) que es utilizado para acceder a distintos bienes públicos (e. g. dádivas, empleo público, obra pública).

Para medir la proximidad entre los votantes y los partidos políticos, se presenta una novedosa metodología que introduce instrumentos en una encuesta aleatoria para estimar la cantidad de conexiones entre votantes y las redes políticas de los distintos partidos. Una vez medida la estructura y el tamaño de las redes políticas, se compara el efecto que tiene la proximidad a un partido y la afinidad ideológica sobre las expectativas distributivas de los votantes. Es decir, se estima la probabilidad reportada por los votantes de percibir bienes públicos en el futuro, condicionada por el tipo de acceso.

Para medir los vínculos entre votantes y miembros partidarios, aprovechamos los recientes avances en el análisis de las redes sociales que utilizan preguntas indirectas tales como «¿A cuántas personas con característica $\mathrm{X}$ conoce...?? para estimar el tamaño de las poblaciones difíciles de contabilizar y para descubrir estructuras sociales a partir de datos individuales (McCarty et al. 2000; Zheng, Salganik y Gelman 2006). Esta metodología permite estudiar redes políticas a partir de información reportada por encuestados individuales, los cuales actúan como observadores y describen propiedades importantes de sus entornos partidarios, así como propiedades de una variedad de redes y categorías sociales.

Este artículo tiene cinco secciones. Las siguientes dos secciones describen el marco teórico, el diseño metodológico y la estrategia de investigación utilizada. La cuarta sección implementa la metodología propuesta al estudio de las redes políticas en Argentina y Chile. La quinta sección evalúa los efectos que tienen la proximidad ideológica y las redes partidarias sobre las expectativas distributivas de los votantes. La sexta sección discute algunas extensiones de esta investigación.

\section{REDES POLÍTICAS Y VÍNCULOS ENTRE LOS PARTIDOS POLÍTICOS Y LOS VOTANTES}

Esta investigación analiza las expectativas distributivas de los votantes, los cuales se encuentran integrados dentro un tejido de redes sociales y políticas. Se reconoce que los votantes tienen preferencias distributivas heterogéneas y que desarrollan expectativas sobre la capacidad de las élites partidarias y de los militantes para entregar bienes tales como dádivas, empleos públicos y obras públicas.

En este trabajo se distingue la demanda distributiva («cuánto quiero») de la expectativa distributiva («cuán probable es que reciba»). Mientras que la demanda distributiva puede ser explicada por características socioeconómicas tales como el ingreso, la clase social o la educación; las expectativas distributivas son moldeadas por experiencias previas en el acceso a beneficios públicos, los cuales dependen de vínculos con redes 
partidarias o de conocimiento previo de las políticas públicas programáticas que proponen las élites.

Se entiende por vínculo, tanto clientelar como programático, las relaciones interpersonales basadas en las expectativas que tienen los votantes de acceder a beneficios públicos a través de redes partidarias o a través de políticas programáticas. Estas expectativas son formadas históricamente por interacciones previas que los votantes tienen con militantes de los distintos partidos así como por el conocimiento que dichos votantes tienen respecto de la oferta programática. Se argumenta que las interacciones personales y el conocimiento sobre aquellas políticas públicas a ser implementadas por los partidos, argumentamos, explican la importancia que los votantes dan a sus redes partidarias o a las propuestas programáticas de los partidos. Es decir, explican la formación de distintas expectativas distributivas.

\section{II.1. Redes politicas y vinculos partidarios}

Investigaciones anteriores sobre los partidos políticos de masa en democracias avanzadas subrayaron el rol esencial de las redes partidarias para explicar los vínculos entre los partidos y los votantes. Esta literatura afirma que los partidos pueden, eventualmente, sustituir a las redes clientelares en la medida en que las conexiones locales se debilitan y la competencia electoral se nacionaliza (Panebianco 1988; Kirchheimer 1966). Los académicos que abogaban por el modelo de partidos responsables, por tanto, asumen que los partidos políticos modernos pueden consolidar posiciones generales en plataformas políticas y que los electores pueden hacer uso de estas propuestas ideológicas para tomar decisiones electorales informadas. Aun cuando es discutible que el modelo de partidos responsables describa correctamente las democracias consolidadas, la expansión de la democracia en países en desarrollo ha sido acompañada por el aumento de los partidos no programáticos que dependen de la distribución de beneficios clientelares para satisfacer las demandas de los votantes no comprometidos ideológicamente. En consecuencia, investigaciones recientes en países en proceso de democratización han despertado el interés sobre las redes políticas y las maquinas partidarias modernas ${ }^{4}$.

Las redes partidarias tienen muchas funciones, las cuales se extienden más allá del reparto de bienes. Por ejemplo, las redes políticas permiten a los políticos reunir información sobre el estado de ánimo, las necesidades y los deseos de los votantes. Las redes partidarias también permiten a los partidos políticos presentar una cara conocida a nivel local, lo cual es necesario para la difusión de sus ideas y objetivos políticos. Sin embargo, en el caso particular de los partidos clientelares, las redes partidarias también sirven

4. La literatura reciente ha puesto el foco en la importancia de las redes para monitorear a los votantes (H. Kitschelt y S. WiLKInSON 2007; S. C. STOKes 2005), identificar simpatizantes (G. W. Cox y M. D. McCubbins 1986; A. Dixit y J. Londregan 1996, 1998; G. W. Cox 2007), movilizar partidarios (S. NiCHTER 2008; M. SZWARCBERG 2012) y coordinar la competencia electoral (G. W. Cox 2007). 
ERNESTO CALVO Y MARÍA VICTORIA MURILLO

CUANDO LOS PARTIDOS POLIITTCOS SE ENCUENTRAN CON SUS VOTANTES: UN ANÁLISIS DE LOS VÍNCULOS

POLÍTICOS A TRAVÉS DE LAS REDES PARTIDARIAS Y LAS EXPECTATIVAS DISTRIBUTIVAS EN ARGENTINA Y CHILE

para identificar a clientes potenciales (Ujhelyi y Calvo 2010), registrar beneficiarios y reducir los costos en la distribución de bienes (Dixit y Londregan 1996; Szwarcberg 2008). Por lo tanto, aun cuando tanto los partidos programáticos como los clientelares se sirven de redes partidarias, tan sólo los partidos clientelares generan expectativas distributivas entre sus votantes que están asociadas a vínculos interpersonales con miembros del partido.

\section{II.2. Las expectativas distributivas de los votantes}

Este trabajo caracteriza las expectativas distributivas de los votantes considerando tres componentes distintos. En primer lugar, los votantes desarrollan diferentes demandas distributivas que son producto de las características socioeconómicas que determinan el valor marginal de los bienes públicos que les gustaría recibir («qué quiero recibir») por parte de los partidos políticos (Cox 2007; Díaz-Cayeros 2008; Dixit y Londregan 1996). Sin embargo, las expectativas distributivas no derivan simplemente de las necesidades de cada votante, sino también de la forma en que los votantes evalúan la capacidad distributiva de los partidos y la probabilidad de efectivamente percibir dichos bienes. Por tanto, en segundo lugar, los votantes desarrollan expectativas distributivas donde la probabilidad de recibir estos beneficios depende de la proximidad ideológica a los partidos políticos. Es decir, donde la distribución focalizada de bienes es el resultado de políticas públicas que favorecen a los votantes en tanto éstos son miembros de un grupo o una categoría social («qué políticas públicas van a implementar»). Finalmente, en tercer lugar, las expectativas de recibir bienes dependen de la importancia que los votantes asignan al acceso a beneficios que dependen de su proximidad física y el acceso que el votante tiene a miembros del partido político («quién lo va a proveer»). Esto es, cuánto peso asigna cada votante a su contacto con los miembros del partido que tienen capacidad para distribuir bienes. En este trabajo, se consideran estos tres componentes como determinantes independientes y exógenos que forman las expectativas distributivas de los votantes 5 .

Es importante destacar nuevamente que la proximidad de las redes partidarias no está solamente basada en las necesidades (need-based). Aun cuando un votante esté ansioso por recibir beneficios privados de un partido político, este votante puede no estar conectado a miembros del partido que podrían proveerle bienes. Por lo tanto, mientras la afinidad ideológica se define por las actitudes de los votantes y las ofertas programáticas de los partidos, los vínculos con miembros del partido dependen del tamaño de la red personal del votante, del alcance de la organización partidaria y de los vínculos que conectan a votantes con los miembros de cada partido.

5. Si bien en algunos contextos puede haber efectos multiplicativos en la entrega de bienes mediante las redes partidarias y las políticas programáticas, la ideología y la proximidad a los miembros del partido no tienen ningún efecto conjunto estadísticamente significativo. 
Se espera que los votantes perciban una red organizacional densa como una ventaja para acceder a beneficios y, a su vez, que los votantes consideren a las redes organizativas del partido como redes de confianza. Por tanto, las expectativas distributivas positivas respecto a los partidos que carecen de capacidad organizativa estarán limitadas a las ofertas programáticas y a la afinidad ideológica. Se espera que las distintas percepciones con respecto a la capacidad organizativa y la afinidad programática de los partidos moldeen las expectativas distributivas de los votantes y sus valoraciones con respecto a cómo acceder a los beneficios. Si los votantes perciben las redes como mecanismos cruciales para acceder a los beneficios, las expectativas distributivas serán explicadas por la cantidad de vínculos que los conectan con los miembros del partido. En cambio, si los votantes perciben diferencias en las capacidades partidarias incrementarán el peso o la importancia que los votantes le otorgarán a la proximidad ideológica, sobre todo, cuando formen sus expectativas futuras sobre la distribución de los beneficios por parte de los partidos. Mientras que el primer proceso fortalecerá los vínculos clientelares entre los partidos políticos y los votantes, el segundo contribuirá a intensificar los vínculos programáticos.

Este marco conceptual hace suponer que la variación en las expectativas distributivas se dará en función de los vínculos políticos que variarán dentro y entre los sistemas políticos. Se espera una variación en los vínculos políticos entre los distintos partidos políticos sobre una misma política porque sus capacidades organizativas para acceder y repartir recursos son percibidas de distinta manera. Se espera también una variación entre los sistemas políticos en función de los desarrollos históricos y las limitantes institucionales. Así, los votantes perciben de manera diferente la capacidad organizativa y los diseños de política por parte de los partidos.

\section{UN MODELO ESTADÍSTICO PARA MEDIR REDES POLÍTICAS}

Para probar el modelo de vínculos clientelares y expectativas distributivas se utiliza una encuesta que mide la forma en que los votantes perciben las distintas capacidades organizativas de los partidos. Esto es, se precisan datos capaces de mostrar el modo en que los votantes evalúan la capacidad organizativa de los partidos y que sirven para formar las expectativas distributivas, clientelares o programáticas. Con este objetivo, se aprovecha una estrategia de encuestas propuesta por primera vez por Christopher McCarthy (2000), que puede ser utilizada para estimar la prevalencia de grupos que se encuentran dispersos en la población.

\section{III.1. Usando datos de la encuesta para medir el tamaño de las redes politicas}

Para medir el tamaño y la estructura de las redes políticas, se usa el diseño de encuesta que considera a cada encuestado de la muestra como un observador que ofrece información sobre la cantidad de vínculos entre el encuestado y las categorías de miembros de los partidos. La encuesta está diseñada con preguntas tales como «¿Cuántos X 
conoces?», pidiendo a cada encuestado que informe sobre cuántos grupos conoce cuya frecuencia en la población es alta («¿Cuántas personas conoces cuyo nombre es Silvia?») y recuentos de grupos cuya frecuencia en la población se intenta estimar («¿Cuántos militantes del Partido Socialista conoces?»). Se aclara al encuestado que conocer a alguien significa que «los conoces, te conocen, y puedes contactarlos por teléfono, carta o en persona y que has tenido contacto por lo menos en los últimos dos años». Un lazo o conexión entre el votante y un miembro del grupo, consecuentemente, implica que hay una relación de mutuo conocimiento y que cierto tipo de interacción ocurrió durante los últimos dos años.

En el diseño de la encuesta, se utiliza información sobre los grupos de conocidos como offsets para escalar los parámetros que miden el tamaño de las redes personales de los encuestados. Por ejemplo, si un encuestado conoce dos Silvias y dado que la relativa prevalencia del nombre Silvia en Argentina es del 0,86\%, una estimación simple de la red personal del encuestado sería aproximadamente $\approx 232$ individuos $\left(N_{P}=\frac{2}{, 0086}\right)$. Con el uso de una batería de preguntas cuya frecuencia es conocida y un modelo estadístico levemente sofisticado, se estima el tamaño de la red personal de cada encuestado ${ }^{6}$.

Una vez estimado el tamaño de la red personal del encuestado, se realiza otra serie de preguntas sobre poblaciones cuya frecuencia es de interés para este artículo, tales como el número de militantes o candidatos de cada partido político relevante. Se usa esta información tanto para estimar la prevalencia de cada grupo en la población como para estimar cuán conectado está el votante a cada grupo. Por ejemplo, si el mismo encuestado conoce a dos Silvias y también a un militante de la UCR (Unión Cívica Radical), se puede medir la relativa prevalencia de los activistas de la UCR como una fracción de la red personal del encuestado $\left(\right.$ Militantes $_{\mathrm{UCR}}=\frac{1}{\text { red personal }}$. Dado que previamente se estimó que la red personal del encuestado es $\approx 232$, se puede entonces estimar que el número de militantes de la UCR es $\approx 0,43$ por ciento de la población argentina (Militantes $\mathrm{UCR}_{\mathrm{UCR}}=\frac{1}{232}$ *poblacion), aproximadamente 166.000 militantes.

La estrategia de la encuesta tiene como ventaja principal la habilidad de recuperar muestras válidas de poblaciones escasamente representadas entre los votantes adultos. Es importante hacer notar que se espera que las categorías de referencia (i. e. Silvia) no estén correlacionadas con las categorías de grupos que se intenta analizar. Al conocer la frecuencia distributiva de la categoría nombre entre las diferentes localidades y

6. Para esta investigación se seleccionaron nombres como categorías de referencia que muestran una variación mínima en los distritos electorales. Esto asegura que las diferencias territoriales estimadas en la frecuencia de las distintas categorías políticas no son una función de la distribución de los nombres en la población. Para seleccionar los nombres utilizados como categorías de referencia, se utilizó la lista completa de los votantes registrados en ambos países (aproximadamente 18 millones de votantes registrados en Argentina y aproximadamente 8 millones de votantes en Chile). Al conocer la distribución de las categorías de referencia de la población, se puede asegurar que las diferencias estimadas en las tasas de prevalencia de los partidarios se miden correctamente. 
ERNESTO CALVO Y MARÍA VICTORIA MURILLO

CUANDO LOS PARTIDOS POLÍTICOS SE ENCUENTRAN CON SUS VOTANTES: UN ANÁLISIS DE LOS VÍNCULOS POLÍTICOS A TRAVÉS DE LAS REDES PARTIDARIAS Y LAS EXPECTATIVAS DISTRIBUTIVAS EN ARGENTINA Y CHILE

poblaciones se puede estimar correctamente el tamaño de la red personal. En cambio, no se espera que la frecuencia de los militantes sea la misma entre los distritos electorales y las categorías socioeconómicas. En consecuencia, estimar correctamente las redes personales permite explorar la variación sustantiva del tamaño, estructura y distribución territorial de las categorías de la red ${ }^{7}$.

\section{III.2. La estrategia estadística: un modelo Poisson corregido por sobredispersión}

Una vez recolectados los datos en bruto para cada subgrupo y para cada encuestado, se requiere un modelo estadístico donde se estimen todos los parámetros de interés. Zheng, Salganik y Gelman (2006) proponen un modelo Poisson corregido por sobredispersión, que estima el tamaño de la red personal y permite explorar la estructura social de los datos. El modelo estima tres series de parámetros: el tamaño relativo de la red personal de cada encuestado, la relativa prevalencia de cada grupo en la población y el parámetro que explora las desviaciones a nivel individual con respecto a las redes personales y los grupos relevantes. El modelo Poisson corregido por sobredispersión usa los recuentos de individuos conocidos por cada encuestado como una variable dependiente y estima tres series de parámetros latentes:

$$
y_{i k} \sim \operatorname{Poisson}\left(e^{\left.\alpha_{i}+\beta_{k}+\delta_{i k}\right)}\right.
$$

donde $\alpha_{i}$ describe el tamaño de la red personal del encuestado $i, \beta_{k}$ describe la prevalencia esperada del grupo $k$ en la población y el parámetro de la sobredispersión $\delta_{i k}$ estima un factor multiplicativo de las desviaciones individuales y grupales de la red personal $\alpha_{i}$ y de la prevalencia de un grupo $\beta_{k}$ (Gelman y Hill 2007). El vector de los parámetros sobredispersos $\delta_{1 k}, \ldots, \delta_{n k}$ provee importante información sobre las desviaciones a nivel individual de la prevalencia de todo el grupo, permitiendo el estudio de la estructura social de la red -cómo las diferentes categorías políticas se relacionan entre sí- por medio de la comparación de la sobredispersión de los parámetros de los individuos para cada grupo. Esto es, se puede identificar si los encuestados con más vínculos con una red partidaria, de acuerdo al tamaño de su red personal, están también asociados con otras actitudes políticas que también se intenta explorar (tal como la distancia ideológica con el partido político) ${ }^{8}$.

7. Un análisis de la estructura territorial de las redes partidarias va más allá del alcance de este artículo. Otros análisis e investigaciones sobre este tema se pueden solicitar a los autores.

8. Es importante tener en cuenta que el modelo estima que el uso de una medida de proximidad normalizada produce efectos similares a los obtenidos usando los datos en bruto. De hecho, los resultados normalizados son conservadores cuando se los compara con los datos sin procesar relativos a los miembros del partido. Sin embargo, las estimaciones que utilizan los datos sin procesar contienen información acerca de las diferencias de tamaño de la red y se espera que estén sesgados. En consecuencia, se considera que las estimaciones correctas deben ser normalizadas tal como lo proponen A. GELMAN y J. HiLL (2007). 
ERNESTO CALVO Y MARÍA VICTORIA MURILLO

CUANDO LOS PARTIDOS POLIITTCOS SE ENCUENTRAN CON SUS VOTANTES: UN ANÁLISIS DE LOS VÍNCULOS

POLÍTICOS A TRAVÉS DE LAS REDES PARTIDARIAS Y LAS EXPECTATIVAS DISTRIBUTIVAS EN ARGENTINA Y CHILE

\section{IDEOLOGía y REDES PARTIDARIAS EN CHILE y ARGENTINA}

Se realizaron encuestas en Chile y Argentina para medir el tamaño y la estructura de las redes partidarias y analizar el efecto de los vínculos programáticos y clientelares sobre las expectativas distributivas de los votantes. Se eligieron Chile y Argentina porque son países con sistemas de partidos caracterizados respectivamente como programáticos y clientelares. Chile y Argentina también permiten controlar por los efectos de otras variables contextuales sobre las cuales se ha teorizado y que afectan los vínculos entre los partidos políticos y los votantes. Ambos países se han democratizado recientemente -1990 y 1983 , respectivamente- y han establecido partidos políticos de masa, que se apoyan sobre etiquetas partidarias claramente identificables y sobre el control para nominar candidatos. Ambos países son presidencialistas, multipartidistas y tienen similares niveles de desarrollo económico y presentan herencias étnicas, religiosas y culturales similares.

Se realizaron dos encuestas representativas a nivel nacional con 2.800 casos, haciendo una muestra con habitantes de ciudades con poblaciones mayores a 40.000 en Chile y 10.000 en Argentina. La encuesta contiene tres módulos que incluyen preguntas diseñadas para medir el (i) tamaño de las redes políticas, (ii) la conducta política de los votantes, (iii) el nivel sociodemográfico de los encuestados.

El primer módulo fue dividido en dos partes. La primera parte contiene preguntas a los encuestados sobre grupos poblacionales con frecuencias conocidas (i. e. nombres, profesiones, eventos sociales) que satisfacen tres criterios: son fácilmente identificables por los votantes y no tienen ambigüedades, reducen la variación entre los distritos electorales y tienen un rango de prevalencia entre $0,1 \%$ y $2 \%$ sobre el total de la población (idealmente cerca del $0,5 \%$ ) para minimizar las distorsiones. Se eligieron estos porcentajes porque los encuestados tienden a mencionar con una baja frecuencia algunas categorías que son muy frecuentes en la población y tienden a mencionar con una alta frecuencia grupos de categorías que no son muy comunes en la población (Gelman y Hill 2007; McCarty et al. 2000). En base a estos criterios, se usan aproximadamente quince preguntas que refieren a categorías sobre las que se conoce de antemano la ratio de prevalencia'.

9. Las categorías de referencia no son las mismas en ambos países con el fin de minimizar los sesgos socioeconómicos y regionales y para ajustar las diferencias en las tasas de prevalencia. En Argentina, los nombres utilizados fueron Silvia, Patricia, Antonio, Francisco y Ángel. Otras categorías incluyen el número de personas que el encuestado sabe que trabajan como policía, como maestro, médico y que reciben programas de trabajo. El encuestado sabe si tuvo un hijo en el último año, se casó en el último año o si tienen una discapacidad física. En Chile, los nombres eran Gladys, Verónica, Marta, Sergio, Jaime, Ricardo, Eduardo, y se preguntó por individuos que trabajaran como profesor, militares, médicos, limpieza, que percibían el programa Chile Solidario, que hubieran tenido un hijo en el último año, muerto en el último año, se casó en el año pasado o tomó su examen de ingreso a la universidad. Los nombres fueron tomados de las listas electorales en cada país, mientras que otras tasas de prevalencia se obtuvieron a partir de datos del censo. 
ERNESTO CALVO Y MARÍA VICTORIA MURILLO

CUANDO LOS PARTIDOS POLÍTICOS SE ENCUENTRAN CON SUS VOTANTES: UN ANÁLISIS DE LOS VÍNCULOS

POLÍTICOS A TRAVÉS DE LAS REDES PARTIDARIAS Y LAS EXPECTATIVAS DISTRIBUTIVAS EN ARGENTINA Y CHILE

La segunda parte de este primer modelo investiga sobre los recuentos de población cuya frecuencia interesa relevar, tales como el número de militantes partidarios de los partidos más importantes y el número de individuos que recibieron dádivas por parte de cada partido. Los siguientes dos modelos se centran en las actitudes, incluyendo el autoposicionamiento ideológico y la posición ideológica de los principales partidos políticos, mientras que el último modelo incluye preguntas sobre las características sociodemográficas que debieran influir en las preferencias distributivas. La encuesta, por consiguiente, permite recuperar las principales variables de interés que pueden medir el impacto de la distancia ideológica, las redes partidarias y las capacidades sobre las expectativas distributivas de Chile y Argentina.

\section{IV.1. Ideología y vínculos entre los partidos políticos y los votantes}

La literatura comparada existente sugiere que los votantes chilenos pueden identificar más fácilmente la ideología de los partidos políticos que los votantes argentinos (Kitschelt et al. 2010). En Chile, los estudiosos reconocen dos coaliciones ideológicas bien definidas que han caracterizado las elecciones desde el plebiscito que precedió a la democratización en 1988. La coalición de centro-izquierda, Concertación de Partidos por la Democracia, que ganó las primeras cuatro elecciones presidenciales desde 1989 y perdió en 2010, incluye a los tres principales partidos: el Partido Socialista (PS), el Partido de la Democracia Cristiana (DC) y el Partido por la Democracia (PPD). La coalición de centro-derecha, Alianza por Chile, incluye dos partidos: Renovación Nacional (RN), heredero del viejo partido conservador Partido Nacional, y Unión Democrática Independiente (UDI), creada en 1987 por aliados al régimen militar de Pinochet (Huneeus 2007). Aunque RN y UDI compitieron en campañas separadas en 2005, lograron coordinarse para la carrera legislativa y presentaron un candidato presidencial conjunto en todas las otras elecciones, incluyendo la elección de 2010, cuando ganaron la presidencia.

Los resultados descriptivos de la encuesta apoyan este punto de vista. Los votantes chilenos de la encuesta pudieron fácilmente identificar la localización ideológica de los partidos en la dimensión izquierda-derecha. Como se muestra en la Figura I, una mayoría identifica al PS en la izquierda del espectro político, con el 70 por ciento de los encuestados que colocan al partido completamente a la izquierda $(40,3$ por ciento) o en la centro-izquierda ( 30 por ciento). El 76 por ciento de los encuestados identifican a la DC en el centro y ubican al PPD en la centro-izquierda, entre el PS y la DC. Los encuestados también identifican claramente al RN y la UDI a la derecha del espectro ideológico. 
ERNESTO CALVO Y MARÍA VICTORIA MURILLO

26 CUANDO LOS PARTIDOS POLÍTICOS SE ENCUENTRAN CON SUS VOTANTES: UN ANÁLISIS DE LOS VÍNCULOS POLÍTICOS A TRAVÉS DE LAS REDES PARTIDARIAS Y LAS EXPECTATIVAS DISTRIBUTIVAS EN ARGENTINA Y CHILE

GRÁFICO I

UBICACIÓN IDEOLÓGICA DECLARADA CON RESPECTO A LOS PRINCIPALES PARTIDOS POLÍTICOS DE CHILE Y ARGENTINA
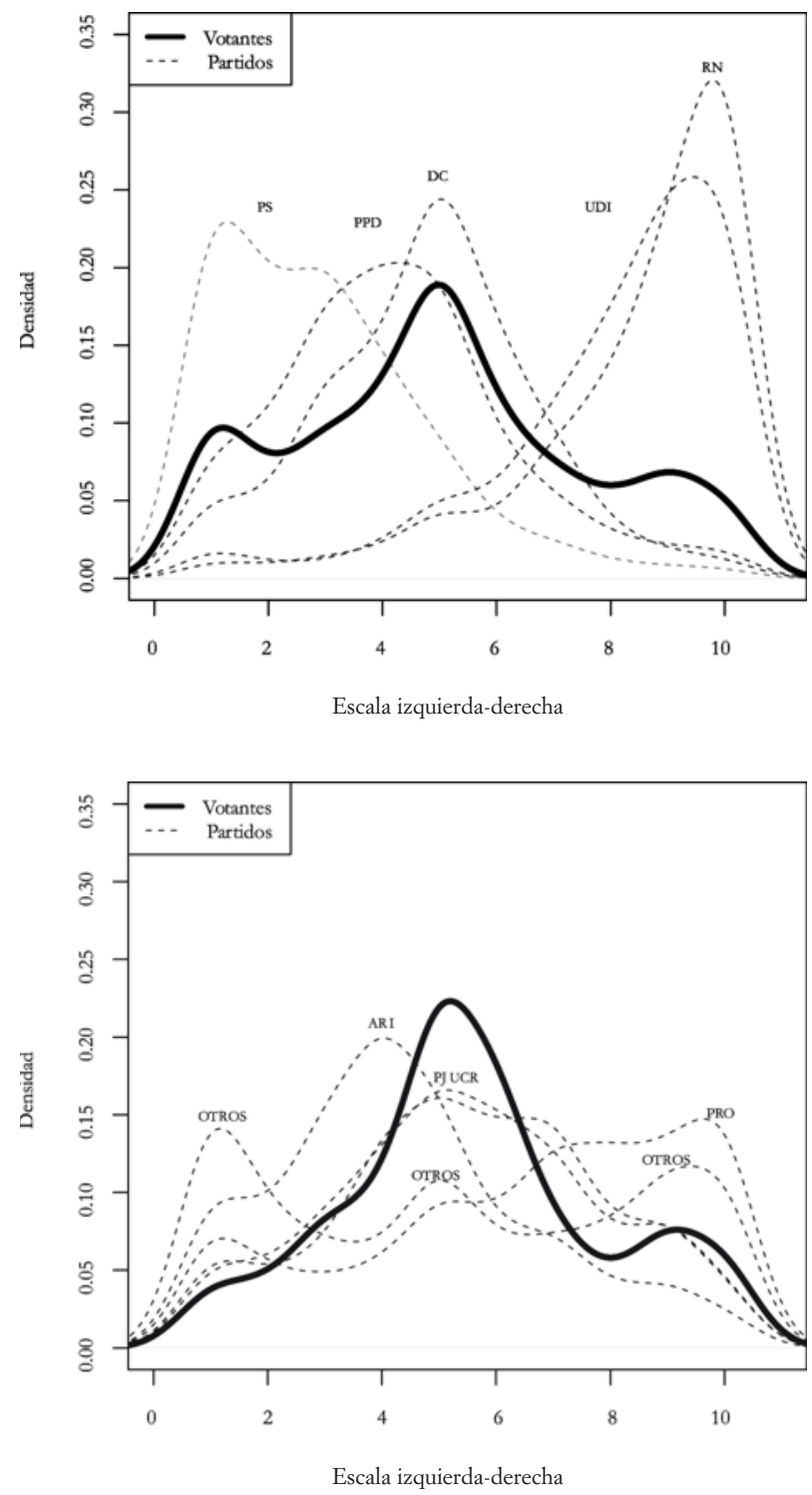

Fuente: Elaboración propia. 
Por el contrario, la encuesta confirma la dificultad de los votantes argentinos para ubicar ideológicamente a los dos principales partidos políticos. En efecto, tanto el partido de la Unión Cívica Radical (UCR), fundado en la década de 1890, como el Partido Justicialista (PJ), creado por Juan D. Perón en la década de 1940, fueron establecidos como partidos catch-all apelando a una amplia coalición multiclasista. Como resultado, ningún partido definió claros nichos ideológicos -aun cuando el PJ haya tenido siempre apoyos de las clases trabajadoras-. La percepción de los votantes que surgen de la encuesta refleja la indefinición de las características ideológicas de los partidos políticos. La frecuencia ideológica modal del PJ, ubicada en una posición de centro, sólo incluye al 21 por ciento de los encuestados. Este número se incrementa al 47 por ciento si se lo combina con las categorías de centro, centro-izquierda y centro-derecha. De manera similar, la frecuencia ideológica modal centrista de la UCR incluye solo al 18,4 por ciento de los encuestados, pero aumenta al 45 por ciento si se incluyen las categorías de centro, centro-izquierda y centro-derecha. La encuesta también mostró un número alto de preguntas sin respuesta relacionadas con la ideología, con un 36 por ciento sin respuesta para el PJ y un 40 por ciento para la UCR. Adicionalmente, en la Figura I se puede observar el comportamiento de dos nuevos partidos que son relevantes políticamente. La Alianza para una República de Iguales (ARI) y Propuesta Republicana (PRO) muestran perfiles ideológicos más definidos, provistos por los votantes de centro-izquierda y de centro-derecha respectivamente. En suma, el comportamiento ideológico de los partidos políticos es más fácilmente identificable para los votantes chilenos que para los argentinos.

\section{IV.2. La organización partidista y los vínculos entre los partidos políticos y los votantes}

Para evaluar el impacto de las redes partidarias sobre las expectativas distributivas de los votantes, se mide el tamaño de la organización partidaria de los principales partidos políticos en ambos países. Se encuentra que el número total de militantes políticos es muy similar, comprendiendo aproximadamente $\approx 1,4 \%$ de la población en Argentina y $\approx 1,2 \%$ en Chile (Tabla I). Sin embargo, los cinco partidos políticos chilenos que se analizan tienen organizaciones de tamaño similar, mientras que la competencia es muy desigual en Argentina. Esto es, las estimaciones de los encuestados con respecto al número de activistas muestra que todos los partidos políticos chilenos tienen aproximadamente la misma cantidad de militantes. El Partido Socialista tiene la red más extensa que incluye aproximadamente a 45.000 activistas $(0,356$ por ciento de la población chilena). Sin embargo, el PS no es mucho más grande que sus competidores, el Demócrata Cristiano (0,299 por ciento), el PPD (0,2 por ciento), la UDI ( 0,2 por ciento), y aun el más pequeño RN ( 0,147 por ciento). Por el contrario, el número de militantes peronistas (PJ) es considerablemente mayor al de otros partidos en Argentina. El PJ tiene alrededor de 291.000 militantes (0,766 por ciento de la población), lo que duplica el número de activistas de la UCR $(\approx 160.000$ o 0,42 por ciento de la población), y ambos, el PJ y la UCR, son varias veces más grandes que 
ERNESTO CALVO Y MARÍA VICTORIA MURILLO

CUANDO LOS PARTIDOS POLIITICOS SE ENCUENTRAN CON SUS VOTANTES: UN ANÁLISIS DE LOS VÍNCULOS

POLIITICOS A TRAVÉS DE LAS REDES PARTIDARIAS Y LAS EXPECTATIVAS DISTRIBUTIVAS EN ARGENTINA Y CHILE

PRO y ARI ${ }^{10}$. En suma, la encuesta realizada para este trabajo sugiere que aunque los partidos políticos en ambos países tienen organizaciones políticas capaces de desplegar ambos tipos de estrategias -programáticas o clientelares-, en Argentina, el PJ y, en menor medida, la UCR, cuentan con grandes ventajas en la búsqueda de electores vis-à-vis sus competidores. Cuando los votantes tienen dificultades para identificar ideológicamente a los partidos, las expectativas distributivas se modelan en función de la capacidad de estas redes para distribuir los beneficios.

La Tabla I también muestra el tamaño estimado de los beneficiarios de dádivas de todos los partidos políticos ${ }^{11}$. Este número es considerablemente mayor para los beneficiarios del partido peronista (0,48 por ciento de la población). El número de individuos que reciben dádivas por parte de los peronistas es dos veces y medio más grande que aquellos que reciben beneficios por parte de los miembros de la UCR $(0,19$ por ciento de la población). El número de beneficiarios de dichos dos partidos es muchas veces superior a los que reciben de otros partidos. Los datos también muestran que las redes se agrandan y se achican lentamente a lo largo del tiempo. En efecto, las redes de la UCR son considerablemente mayores a las esperadas dada su débil performance electoral desde aproximadamente el año 2001. Tal como describió el senador de la UCR y candidato a presidente Leopoldo Moreau:

... el radicalismo es un partido que mantiene su estructura. Porque es cierto que cada pueblo tiene su curita. Digamos, es una red que se desarrolló a lo largo de más de cien años, no puede desaparecer de la noche a la mañana. Puede tener altibajos, avances y retrocesos, pero no puede desaparecer de la noche a la mañana ${ }^{12}$.

Las redes partidarias en Chile tienen tamaños similares. El PS muestra una pequeña ventaja en el número de militantes y la UDI muestra una pequeña ventaja en el número de beneficiarios de dádivas. Los hallazgos de este trabajo están en concordancia con investigaciones recientes que describen a la UDI como el partido chileno que más bienes clientelísticos distribuye durante las elecciones (Luna 2010). Aun así, la red distributiva de la UDI es significativamente menor que aquella del PJ y la UCR. Consecuentemente, la ratio entre redes partidarias y redes distributivas en Chile es significativamente menor que la observada para la UCR, y especialmente para el PJ en el caso de Argentina.

10. En Argentina, todas las referencias al Partido Político Provincial (PPP) en las tablas y los gráficos se corresponden con el principal partido provincial de cada provincia del encuestado. Sin embargo, se agrupan todas estas partes juntas a pesar de que existan grandes diferencias entre ellos, y por ello se intenta no sacar conclusiones de estos hallazgos.

11. Esta medida se construye como la de los militantes del partido tomando a los encuestados en calidad de observadores y por lo tanto reduce el sesgo de la característica de la autopercepción con respecto a las medidas sobre la distribución clientelar.

12. Leopoldo Moreau, entrevista personal con los autores, 2009. 
TABLA I

ÍNDICE DE PREDOMINIO DE GRUPO POLÍTICO COMO PORCENTAJE DE LA POBLACIÓN Y EN NÚMEROS ABSOLUTOS EN CHILE Y ARGENTINA

N RED POLÍTICA

Candidato PS 26.

Candidato DC

Candidato PJ

Candidato UCR

125.376

Militante DC

21.074

Militante UCR

290.930

69.532

Candidato PPD 15.077

Militante PPD $\quad 30.257$

Candidato UDI 16.022

Candidato AR

159.684

Militante ARI

9.908

Candidato PRO

21.463

Militante UDI $\quad 30.031$

Candidato RN $\quad 13.130$

Militante RN

Militante PRO

4.257

Candidato PPP

10.853

Militante PPP

18.060

Bienes PS

22.283

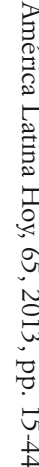

Bienes DC

Bienes PJ

185.052

Bienes PPD

11.614

Bienes UCR

72.472

Bienes UDI

23.377

Bienes ARI

10.074

Bienes RN

Bienes PRO

6.535

Fuente: Elaboración propia.

Bienes PPP

23.893

RED POLÍTICA \%

Candidato PS

Militante PS

Candidato DC

Militante DC

Candidato PPD

Militante PPD

Candidato UDI

Militante UDI

Candidato RN

Militante RN

Bienes PS

Bienes DC

Bienes PPD

Bienes UDI

Bienes RN

0,177
0,356
0,139
0,299
0,1
0,2
0,106
0,199
0,087
0,147
0,114
0,129
0,077
0,155
0,109

RED POLÍTICA

$\%$

Candidato PJ $\quad 0,33$

Militante PJ

Candidato UCR $\quad 0,183$

Militante UCR $\quad 0,42$

Candidato ARI $\quad 0,026$

Militante ARI $\quad 0,056$

Candidato PRO 0,011

Militante PRO $\quad 0,029$

Candidato PPP $\quad 0,048$

Militante PPP $\quad 0,108$

Bienes PJ $\quad 0,487$

Bienes UCR $\quad 0,191$

Bienes ARI $\quad 0,027$

Bienes PRO 0,017

Bienes PPP

0,063 
ERNESTO CALVO Y MARÍA VICTORIA MURILLO

CUANDO LOS PARTIDOS POLIITTCOS SE ENCUENTRAN CON SUS VOTANTES: UN ANÁLISIS DE LOS VÍNCULOS

POLÍTICOS A TRAVÉS DE LAS REDES PARTIDARIAS Y LAS EXPECTATIVAS DISTRIBUTIVAS EN ARGENTINA Y CHILE

\section{IV.3. Partidos políticos y prácticas distributivas}

Este trabajo postula que el tipo de vínculo entre los partidos políticos y los votantes explica las diferencias en las expectativas distributivas de los electores. En la sección anterior se detallaron una serie de similitudes internas en cuanto al tamaño y la estructura de las redes partidarias tanto dentro como entre los países. En particular, se ha mostrado el relativo equilibrio de la competencia entre los principales partidos políticos chilenos en contraste con el amplio alcance de la UCR y especialmente del PJ y su red de activistas en Argentina. Adicionalmente, existen diferencias significativas en la forma en que los votantes perciben la posición programática ideológica de los partidos políticos. Los resultados de la encuesta muestran las dificultades de los votantes argentinos para ubicar al PJ y la UCR en el espectro ideológico -en contraste con los otros dos partidos nuevos-. En cambio, los encuestados chilenos pueden situar más fácilmente a los diferentes partidos en el espectro ideológico.

Además de las diferencias organizativas e ideológicas entre los partidos políticos, las variaciones institucionales entre los países pueden definir la habilidad de los políticos de apropiarse de los recursos para repartirlos a sus electores. Esto influye en la formación de las expectativas distributivas de los electores. Las limitaciones institucionales en Chile hacen que los partidos chilenos estén más regulados y enfrenten dificultades significativas en asignar bienes financiados por fondos públicos a través de sus redes políticas ${ }^{13}$. Más aun, las reglas del servicio civil en Chile permiten que los votantes sepan que los empleos del sector público son bienes exclusivos cuyo acceso no depende de las redes partidarias (Bau Aedo 2005; Rehren 2000). Como resultado de ello se espera que los votantes chilenos, en promedio, reporten una menor expectativa de recibir bienes clientelares a través de las redes partidarias.

Contrariamente, Guiraudi (2007) y Weitz-Shapiro $(2006,2008)$ aportan evidencia de la significativa discrecionalidad por parte de los funcionarios públicos argentinos en la distribución de ayudas por desempleo y asistencia de alimentos. De manera similar, los empleos públicos en Argentina están sumamente politizados y dependen de los contactos políticos. Así, las percepciones de los votantes sobre la probabilidad de obtener un empleo en el sector público aumentan con la proximidad a las redes partidarias (Szwarcberg 2008; Kemahlioglu 2006). Estas diferentes prácticas en la implementación de la distribución de los bienes públicos debería reforzar la percepción de los votantes sobre el rol de las redes en acceder a los beneficios.

En suma, se espera una variación tanto entre como dentro de los sistemas políticos. A nivel nacional, se espera que las limitaciones institucionales formen las expectativas distributivas de los votantes. Los encuestados chilenos disminuirán el peso del papel de las redes partidarias en comparación con los encuestados en Argentina, que reconocen su importancia. Adicionalmente, se espera que los votantes chilenos de cualquiera de los cinco partidos desarrollen expectativas distributivas de acuerdo con la

13. J. P. LunA y R. MARDONES (2009) han documentado la restringida discreción de los políticos chilenos en la distribución focalizada de políticas sociales. 
proximidad ideológica, las cuales a su vez se encuentra constreñidas por las redes y reforzadas por las experiencias políticas previas. Mientras tanto, se espera que los votantes argentinos confíen menos en la afinidad ideológica y más en las conexiones dentro de las redes partidarias cuando informan sus expectativas distributivas con respecto al PJ y a la UCR. Contrariamente, los vínculos ideológicos deberían ser más útiles en definir las expectativas en los casos de PRO y de ARI. A continuación se ofrecen pruebas empíricas de estas expectativas basadas en la metodología descrita previamente.

\section{VÍNCULOS POLÍtTICOS Y EXPECTATIVAS DISTRIBUTIVAS DE LOS VOTANTES}

En esta sección se testea la forma en que la afinidad ideológica y la proximidad con los miembros del partido influye sobre las expectativas de los votantes en recibir bienes exclusivos. Se toman tres preguntas de la encuesta en las cuales se pregunta a los encuestados sobre la probabilidad de recibir prebendas tales como ropa, comida u otros beneficios materiales (clientelismo); aceptar un trabajo en el sector público (patronazgo); o recibir una mayor inversión pública para su comunidad (pork) si un partido determinado gana las elecciones. La primera pregunta pedía a los encuestados que indicasen, en una escala del uno al diez, «¿qué tan probable sería que, después de ganar las elecciones, el [Partido j] le provea ropa, comida y otros beneficios materiales?». Una pregunta similar decía «¿qué tan probable sería que, después de ganar una elección, el [Partido j] le provea un empleo en el sector público?» (patronazgo). Finalmente, la tercera pregunta establecía «¿qué tan probable sería que, después de ganar las elecciones, el [Partido j] invierta en obras públicas requeridas por su comunidad?» (pork). Con el fin de controlar el sesgo de los individuos en relación con la distribución de estos bienes privados, se incluye una pregunta sobre la postura subjetiva de los mismos con respecto a la distribución de los bienes. Las respuestas a esta pregunta se utilizan como variable dependiente. Con dichas respuestas se hacen modelos de regresión beta por cada partido político y se estima si la distancia o proximidad ideológica con los miembros del partido explican la percepción sobre la propensión a distribuir bienes, empleos u obra pública $^{14}$.

Se prueba el efecto de las dos principales variables independientes: (i) la relativa proximidad de los votantes con los militantes del partido y (ii) la autopercepción reportada por los encuestados sobre la distancia ideológica entre los votantes y los partidos. La proximidad de los votantes con los militantes políticos se mide a través de un número relativo de vínculos entre los votantes y los militantes partidarios. Esto se define como una medida relativa y no absoluta de la proximidad, dado que se ajusta por la diferencia en el tamaño de la red personal de cada encuestado y el grupo predominante de la población de referencia. Dicha información es capturada por la sobredispersión del parámetro $\delta_{i, k}^{\prime}$ de la ecuación (1), que mide las desviaciones geométricas estándar respecto al número promedio de relaciones ajustadas por el tamaño de la red personal

14. También se estima que los modelos alternativos que usan mínimos cuadrados ordinarios (OLS) y Order Probit obtienen resultados similares. 
del encuestado y la prevalencia de cada grupo partidario dentro de la población. Por ejemplo, una estimación de la proximidad del encuestado respecto a los militantes peronistas de $\delta_{i, P J}^{\prime} \exp (0,69)=2$ indica que el encuestado conoce al doble de activistas del partido peronista de lo que se hubiese esperado dada la prevalencia del grupo y el tamaño de la red personal del encuestado. También se incluye como variable independiente el parámetro que mide la proximidad entre cada encuestado y los candidatos de cada partido. Aun cuando todos los modelos usan una distancia normalizada entre cada red de partidos, los resultados sin procesar producen efectos similares ${ }^{15}$.

$\mathrm{Al}$ testear los determinantes de las expectativas distributivas de los votantes, se miden los efectos de las dos principales series de variables. La primera variable independiente testea los efectos que tienen la proximidad a las redes de activistas y candidatos sobre las expectativas distributivas. Se espera una relación positiva cuando una alta proximidad con las redes partidarias incrementa la percepción de probabilidad de recibir bienes. Sin embargo, se anticipa que este efecto es más fuerte para los encuestados en Argentina, y en particular para las redes partidarias del PJ y la UCR. Se espera que la proximidad a una red sea un predictor más débil de las expectativas distributivas entre los votantes chilenos y para el caso de los nuevos partidos políticos argentinos con organizaciones menos extensas.

La segunda variable independiente mide la distancia ideológica entre los encuestados y los partidos políticos. Esta variable se mide tomando la distancia absoluta entre la autoubicación ideológica del encuestado y la ubicación de cada partido: Ideología $(\mathrm{k})=\left|X_{i}-S_{k}\right|$. Se espera que la distancia ideológica tenga un efecto negativo en las expectativas distributivas de los encuestados, donde los votantes más distantes respecto a la ubicación ideológica del partido reportada por los encuestados presenten bajas expectativas de recibir beneficios. Sin embargo, se espera que este efecto sea más fuerte en Chile y para el caso de los partidos pequeños en Argentina debido a las mencionadas diferencias en la importancia de la ideología.

También se agregan una serie de variables independientes como variables de control que determinan los rendimientos marginales de los encuestados en la distribución de bienes exclusivos. Se incluye una batería de variables personales específicas para medir el tamaño de la red personal del encuestado (ln), el nivel educativo de los encuestados, el estatus socioeconómico (ln) y el género. Se usa la educación y el ingreso para identificar las características socioeconómicas de los encuestados. Los niveles educativos y los ingresos bajos aumentan la utilidad marginal de los beneficios recibidos. En concordancia con las investigaciones existentes, se espera que el efecto de la educación en la utilidad de los empleos del sector público aumente en los niveles más bajos y decrezca en los niveles altos. No están claros los efectos de la educación en las expectativas sobre los beneficios esperados en el caso de una mayor inversión en obra pública. No hay expectativas teóricas particulares acerca de la edad, el sexo o el nivel de gregarismo político del entrevistado.

15. Los resultados de todos los modelos que usan los datos sin procesar reportados por los encuestados están a disposición de los interesados. 
Más allá de los pequeños beneficios para cada encuestado en la muestra, se esperan evaluaciones sociotrópicas con respecto a la conveniencia de distribuir bienes. Debido a que los votantes tienen diferentes percepciones sobre cuán apropiado es que un partido distribuya dádivas, empleos públicos y obra pública, se incluye una variable independiente que pide a los encuestados que expresen sus sentimientos -positivos o negativos- con respecto a la distribución de donaciones, empleos públicos y obra públi$\mathrm{ca}^{16}$. Para evaluar el impacto de las diferencias institucionales en la distribución de los fondos públicos mencionados anteriormente, se incluye la proximidad de los encuestados en la red de beneficiarios a través de dos programas sociales con diseños similares y con una implementación descentralizada: Chile Solidario (Chile) y Jefes y Jefas (Argentina). Al tratarse de programas de transferencias de ingreso, se espera que tengan un efecto positivo sobre las expectativas distributivas en relación con las donaciones. Debido a las diferencias en el reparto de fondos públicos, se espera que estos efectos sean significativos en Argentina pero no en Chile. También se controla por la proximidad de los individuos a los miembros del partido que participan en las internas. Se espera que la proximidad de los individuos involucrados en las primarias tenga un efecto positivo en las expectativas distributivas porque la literatura sobre Argentina mide el clientelismo con la participación en las primarias dado que es más sencillo monitorear la concurrencia allí antes que en los comicios generales cuando el voto es obligatorio.

\section{RESULTADOS EMPÍRICOS}

Las Tablas II y III presentan las estimaciones de los modelos de regresión beta para Chile y Argentina. Todos los coeficientes de los modelos de regresión beta pueden ser interpretados como coeficientes OLS, con una unidad de variación en la variable independiente que lleva hacia el cambio estimado del coeficiente con respecto a la probabilidad reportada de recibir dádivas, empleos públicos o fondos públicos para la comunidad ${ }^{17}$. Por ejemplo, el aumento en un punto en la distancia ideológica del PS en Chile resultaría en una disminución del 3,46\% en la probabilidad de recibir bienes de ese partido. En ambas tablas, la primera serie de cinco columnas describe las estimaciones del modelo que miden las expectativas de recibir donaciones de cada uno de los principales partidos políticos; la segunda serie de cinco columnas describe la expectativa de recibir el ofrecimiento de un empleo público; y la tercera serie de cinco columnas describe la expectativa de que los partidos inviertan en obra pública en la comunidad. Se discuten los resultados comparando las expectativas distributivas de cada tipo de beneficio en ambos países con el fin de evaluar el peso de los vínculos programáticos y clientelares.

16. En cada modelo la variable independiente que mide la percepción positiva o negativa sobre la intención redistributiva de los partidos coincide con el tipo de bienes descriptos en la variable dependiente.

17. Los modelos de regresión beta también incluyen una estimación de la precisión (phi), que explica la variación total en la variable dependiente. Si se especifica, la precisión puede ser explicada por un conjunto de covariables. En las especificaciones, se estima un solo parámetro de precisión por modelo sin covariables. 
TABLA II

EXPECTATIVAS DISTRIBUTIVAS, IDEOLC

ÍA Y PROXIMIDAD A LAS REDES PARTIDARIAS EN CHILE

Nota: Modelo de regresión Beta con rango de variable dependiente de 0 (improbable) a 1 (extremadamente probable).

\begin{tabular}{|c|c|c|c|c|c|c|c|c|c|c|c|c|c|c|c|}
\hline & \multicolumn{5}{|c|}{ «Espera recibir Bienes, Dinero u otros incentivos materiales» } & \multicolumn{5}{|c|}{ «Espera recibir un Trabajo en el Sector Público» } & \multicolumn{5}{|c|}{ «Espera recibir la Obra Pública que requiere la Comunidad» } \\
\hline & PS & $\mathrm{DC}$ & PPD & UDI & RN & PS & $\mathrm{DC}$ & PPD & UDI & $\mathrm{RN}$ & PS & C & PPD & DI & RN \\
\hline Constante & $\begin{array}{l}0,8824 \\
(1,750)\end{array}$ & $\begin{array}{c}-0,5458 \\
(1,748)\end{array}$ & $\begin{array}{c}-0,0905 \\
(1,738)\end{array}$ & $\begin{array}{l}0,6315 \\
(1,858)\end{array}$ & $\begin{array}{l}1,2317 \\
(1,846)\end{array}$ & $\begin{array}{l}0,9566 \\
(1,734)\end{array}$ & $\begin{array}{l}0,3669 \\
(1,718)\end{array}$ & $\begin{array}{l}0,8525 \\
(1,695)\end{array}$ & $\begin{array}{c}-0,9143 \\
(1,740)\end{array}$ & $\begin{array}{l}-0,817 \\
(1,718)\end{array}$ & $\begin{array}{l}0,7732 \\
(1,891)\end{array}$ & $\begin{array}{l}-0,798 \\
(1,821)\end{array}$ & & $\begin{array}{l}-1,4121 \\
(1,864)\end{array}$ & $\begin{array}{c}-1,6033 \\
(1,865)\end{array}$ \\
\hline Ideología & $\begin{array}{c}-0,0346^{* * * *}- \\
\quad(0,007)\end{array}$ & $\begin{array}{l}-0,0354 * \% *- \\
\quad(0,008)\end{array}$ & $\begin{array}{c}-0,0212 * * * * \\
(0,008)\end{array}$ & $\begin{array}{r}-0,0032 \\
(0,007)\end{array}$ & $\begin{array}{r}-0,0055 \\
(0,007)\end{array}$ & $\begin{array}{l}-0,0424 * * * *- \\
\quad(0,007)\end{array}$ & 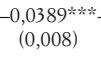 & $\begin{array}{l}-0,0266 * * * ; \\
\quad(0,007)\end{array}$ & $\begin{array}{c}-0.0191 \text { * } \\
(0,007)\end{array}$ & $\begin{array}{c}-0,0188^{* * * *} \\
(0,007)\end{array}$ & $\begin{array}{c}-0,0531^{* * * *} \\
(0,008)\end{array}$ & $\begin{array}{c}-0,047^{* * * *}- \\
(0,008)\end{array}$ & $\begin{array}{c}-0,0384 * * * \% \\
\quad(0,008)\end{array}$ & $\begin{array}{l}-0,0301 * * * *-1 \\
\quad(0,007)\end{array}$ & $\begin{array}{c}-0,0306^{* * * * *} \\
(0,007)\end{array}$ \\
\hline Red de candidatos & $\begin{array}{l}0,0084 \\
(0,036)\end{array}$ & $\begin{array}{l}0,0068 \\
(0,038)\end{array}$ & $\begin{array}{l}0,0521 \\
(0,045)\end{array}$ & $\begin{array}{c}-0,0174 \\
(0,047)\end{array}$ & $\begin{array}{r}-0,0553 \\
(0,050)\end{array}$ & $\begin{array}{l}0,0182 \\
(0,035)\end{array}$ & $\begin{array}{l}0,0076 \\
(0,037)\end{array}$ & $\begin{array}{c}-0,0187 \\
(0,044)\end{array}$ & $\begin{array}{c}-0,0108 \\
(0,043)\end{array}$ & $\begin{array}{c}-0,0446 \\
(0,045)\end{array}$ & $\begin{array}{l}0,0391 \\
(0,038)\end{array}$ & $\begin{array}{l}0,0171 \\
(0,039)\end{array}$ & $\begin{array}{c}0,1125^{* *} \\
(0,046)\end{array}$ & $\begin{array}{c}0,1177^{* *} \\
(0,046)\end{array}$ & $\begin{array}{c}0,08^{*} \\
(0,048)\end{array}$ \\
\hline Red de activistas & $\begin{array}{l}0,0348 \\
(0,027)\end{array}$ & $\begin{array}{l}0,0219 \\
(0,028)\end{array}$ & & $\begin{array}{l}0,0478 \\
(0,034)\end{array}$ & & & $\begin{array}{l}0,0375 \\
(0,028)\end{array}$ & $\begin{array}{c}0,0972 \ldots * * \\
(0,030)\end{array}$ & $\begin{array}{c}-0,0023 \\
(0,032)\end{array}$ & $\begin{array}{c}0,033 \\
(0,035)\end{array}$ & & $\begin{array}{r}-0,0059 \\
(0,029)\end{array}$ & $\begin{array}{c}0,0809^{* * *} \\
(0,033)\end{array}$ & $\begin{array}{c}0,0894 * * * * \\
(0,033)\end{array}$ & \\
\hline Edad & $\begin{array}{r}-1,1127 \\
(0,943)\end{array}$ & $\begin{array}{l}-0,3609 \\
(0,941)\end{array}$ & & & & & & & & & & & & & \\
\hline $\mathrm{Edad}^{\wedge} 2$ & $\begin{array}{l}0,1243 \\
(0,127)\end{array}$ & $\begin{array}{l}0,0256 \\
(0,126)\end{array}$ & $\begin{array}{l}0,0511 \\
(0,126)\end{array}$ & $\begin{array}{l}0,1102 \\
(0,134)\end{array}$ & $\begin{array}{l}0,15 \\
(0,13\end{array}$ & $\begin{array}{l}0,1049 \\
(0,125)\end{array}$ & $\begin{array}{l}0,0942 \\
(0,124)\end{array}$ & $\begin{array}{l}0,1107 \\
(0,123)\end{array}$ & $\begin{array}{l}-0,0014 \\
(0,126)\end{array}$ & $\begin{array}{l}0,0 \\
(0,\end{array}$ & & $\begin{array}{c}0,002 \\
(0,131)\end{array}$ & & & \\
\hline Mujer & & & & $\begin{array}{c}0,034 \\
(0,037)\end{array}$ & & & & & & & & & & & \\
\hline sonal & $\begin{array}{l}0,0463 * \\
(0,024)\end{array}$ & $\begin{array}{l}0,0405^{*} \\
(0,024)\end{array}$ & & & $\begin{array}{c}0,019 \\
(0,025)\end{array}$ & $\begin{array}{c}0,0543 * * \\
(0,024)\end{array}$ & $\begin{array}{c}0,0668 * * * * \\
(0,024)\end{array}$ & & & & $\begin{array}{c}0,0528 * * \\
(0,026)\end{array}$ & $\begin{array}{c}0,0691 * * * * \\
(0,025)\end{array}$ & $\begin{array}{c}0,0688 * * * * \\
(0,025)\end{array}$ & $\begin{array}{c}0,0687 * * * * \\
(0,025)\end{array}$ & \\
\hline Red «Chile Solidario» & "》 $-0,032$ & $\begin{array}{l}-0,0271 \\
(0,022)\end{array}$ & $\begin{array}{r}-0,0243 \\
(0,021)\end{array}$ & $\begin{array}{c}-0,0208 \\
(0,023)\end{array}$ & $\begin{array}{l}-0,027 \\
(0,023)\end{array}$ & $\begin{array}{c}-0,0429^{* * *} \\
(0,022)\end{array}$ & $\begin{array}{r}-0,0181 \\
(0,021)\end{array}$ & $\begin{array}{c}-0,0238 \\
(0,021)\end{array}$ & $\begin{array}{l}-0,0058 \\
(0,021)\end{array}$ & $\begin{array}{r}-0,0 \\
(0,0\end{array}$ & $\begin{array}{c}-0,0948^{* * * *} \\
(0,023)\end{array}$ & $\begin{array}{c}-0,0507 * * \\
(0,022)\end{array}$ & $\begin{array}{l}-0,0884 * * * \\
\quad(0,023)\end{array}$ & $\begin{array}{r}-0,0365 \\
(0,023)\end{array}$ & \\
\hline Red de Primarias & $\begin{array}{l}0,0017 \\
(0,025)\end{array}$ & $\begin{array}{l}0,0134 \\
(0,025)\end{array}$ & $\begin{array}{l}0,0011 \\
(0,024)\end{array}$ & $\begin{array}{l}0,0337 \\
(0,025)\end{array}$ & $\begin{array}{c}0,039 \\
(0,025)\end{array}$ & $\begin{array}{l}0,0397 \\
(0,025)\end{array}$ & $\begin{array}{l}0,0327 \\
(0,024)\end{array}$ & $\begin{array}{c}0,0456 * * \\
(0,023)\end{array}$ & $\begin{array}{l}0,0102 \\
(0,024)\end{array}$ & $\begin{array}{l}0,0108 \\
(0,023)\end{array}$ & $\begin{array}{c}0,0846^{* * * * *} \\
(0,027)\end{array}$ & $\begin{array}{c}0,1074 * * * * \\
(0,025)\end{array}$ & $\begin{array}{c}0,0684 * \cdots * * \\
(0,025)\end{array}$ & $\begin{array}{l}0,0188 \\
(0,025)\end{array}$ & $\begin{array}{l}0,0293 \\
(0,025)\end{array}$ \\
\hline $\begin{array}{l}\text { Educación } \\
\text { Secundaria }\end{array}$ & $\begin{array}{r}-0,0055 \\
(0,011)\end{array}$ & $\begin{array}{l}0,0112 \\
(0,011)\end{array}$ & $\begin{array}{l}0,0144 \\
(0,011)\end{array}$ & $\begin{array}{c}0,0243 * * \\
(0,011)\end{array}$ & $\begin{array}{l}0,0194 * \\
(0,011)\end{array}$ & $\begin{array}{l}0,0041 \\
(0,011)\end{array}$ & $\begin{array}{c}0,015 \\
(0,011)\end{array}$ & $\begin{array}{l}0,0178^{*} \\
(0,010)\end{array}$ & $\begin{array}{l}0,0175^{*} \\
(0,011)\end{array}$ & $\begin{array}{l}0,0172 \\
(0,011)\end{array}$ & $\begin{array}{l}0,0186 \\
(0,011)\end{array}$ & $\begin{array}{c}0,0212^{*} \\
(0,011)\end{array}$ & $\begin{array}{c}0,0249 * * \\
(0,011)\end{array}$ & $\begin{array}{c}0,0297 * \ldots * * \\
(0,011)\end{array}$ & $\begin{array}{c}0,0269 * * \\
(0,011)\end{array}$ \\
\hline $\begin{array}{l}\text { Nivel Socio- } \\
\text { económico alto }\end{array}$ & $\begin{array}{r}-0,0335 \\
(0,044)\end{array}$ & $\begin{array}{c}-0,0219 \\
(0,044)\end{array}$ & $\begin{array}{l}-0,021 \\
(0,044)\end{array}$ & $\begin{array}{c}-0,0315 \\
(0,046)\end{array}$ & $\begin{array}{c}-0,0263 \\
(0,046)\end{array}$ & $\begin{array}{l}0,0005 \\
(0,044)\end{array}$ & $\begin{array}{c}0,012 \\
(0,043)\end{array}$ & & & & $\begin{array}{l}0,0584 \\
(0,047)\end{array}$ & & & & \\
\hline $\begin{array}{l}\text { Nivel Socio- } \\
\text { económico medio }\end{array}$ & $\begin{array}{l}0,0067 \\
(0,056)\end{array}$ & $\begin{array}{l}-0,0587 \\
(0,056)\end{array}$ & $\begin{array}{c}-0,0636 \\
(0,056)\end{array}$ & $\begin{array}{c}-0,1063 * \\
(0,060)\end{array}$ & $\begin{array}{r}-0,0597 \\
(0,059)\end{array}$ & $\begin{array}{l}-0,0048 \\
(0,056)\end{array}$ & $\begin{array}{l}-0,047 \\
(0,055)\end{array}$ & $\begin{array}{r}-0,0123 \\
(0,055)\end{array}$ & $\begin{array}{l}-0,0251 \\
(0,056)\end{array}$ & $\begin{array}{c}-0,0243 \\
(0,055)\end{array}$ & $\begin{array}{l}0,0015 \\
(0,060)\end{array}$ & $\begin{array}{r}-0,0083 \\
(0,058)\end{array}$ & $\begin{array}{l}0,0031 \\
(0,059)\end{array}$ & $\begin{array}{c}0,1311^{* *} \\
(0,059)\end{array}$ & $\begin{array}{c}0,1393 * * \\
(0,059)\end{array}$ \\
\hline Bajo status & $\begin{array}{l}0,1399^{*} \\
(0,074)\end{array}$ & $\begin{array}{l}0,0943 \\
(0.073)\end{array}$ & $\begin{array}{l}0,1297^{*} \\
(0.073)\end{array}$ & $\begin{array}{l}0,0814 \\
(0.078)\end{array}$ & $\begin{array}{l}0,0873 \\
(0,078)\end{array}$ & $\begin{array}{l}0,0938 \\
(0,073)\end{array}$ & $\begin{array}{l}0,0891 \\
(0,072)\end{array}$ & $\begin{array}{l}0,1093 \\
(0,071)\end{array}$ & $\begin{array}{l}0,0867 \\
(0,073)\end{array}$ & $\begin{array}{l}0,0722 \\
(0,073)\end{array}$ & $\begin{array}{c}0,012 \\
(0,080)\end{array}$ & $\begin{array}{l}0,0539 \\
(0,076)\end{array}$ & $\begin{array}{l}0,0848 \\
(0,077)\end{array}$ & $\begin{array}{l}0,1461^{*} \\
(0,078)\end{array}$ & $\begin{array}{c}0,1469^{* *} \\
(0,078)\end{array}$ \\
\hline $\begin{array}{c}\text { Valoración positiva } \\
\text { de la distribución }\end{array}$ & $\begin{array}{c}0,1181 * * * * \\
(0,014)\end{array}$ & $\begin{array}{c}0,1085^{* * * *} \\
(0,014)\end{array}$ & $\begin{array}{c}0,1175^{* * * *} \\
(0,014)\end{array}$ & $\begin{array}{c}0,0755^{* \ldots * * *} \\
(0,015)\end{array}$ & $\begin{array}{c}0,0664 * \% * \% \\
(0,015)\end{array}$ & $\begin{array}{c}0,115^{* * * *} \\
(0,014)\end{array}$ & $\begin{array}{c}0,0807 * * * * \\
(0,011)\end{array}$ & $\begin{array}{c}0,0835 * * * * \\
(0,011)\end{array}$ & $\begin{array}{c}0,0742^{* * * * *} \\
(0,011)\end{array}$ & $\begin{array}{c}0,0755^{* * * *} \\
(0,011)\end{array}$ & $\begin{array}{c}0,1242 * * * \\
(0,012)\end{array}$ & $\begin{array}{c}0,1195^{* * * *} \\
(0,011)\end{array}$ & $\begin{array}{c}0,1238^{* * * * *} \\
(0,011)\end{array}$ & $\begin{array}{c}0,0968^{* * * *} \\
(0,011)\end{array}$ & 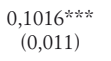 \\
\hline N) & $\begin{array}{c}0,0338^{* * * * * *} \\
(0,010)\end{array}$ & $\begin{array}{c}0,0252^{* * *} \\
(0,010)\end{array}$ & $\begin{array}{c}0,0206 * * * \\
(0,010)\end{array}$ & $\begin{array}{c}0,0289 * * * * \\
(0,011)\end{array}$ & $\begin{array}{l}0,0203^{*} \\
(0,011)\end{array}$ & $\begin{array}{c}0,0266^{* * * * * *} \\
(0,010)\end{array}$ & $\begin{array}{l}0,0174^{*} \\
(0,010)\end{array}$ & $\begin{array}{l}0,0128 \\
(0,010)\end{array}$ & $\begin{array}{l}0,0091 \\
(0,010)\end{array}$ & $\begin{array}{l}0,0169^{* *} \\
(0,010)\end{array}$ & $\begin{array}{c}-0,0078 \\
(0,011)\end{array}$ & $\begin{array}{c}-0,0091 \\
(0,010)\end{array}$ & $\begin{array}{c}-0,0061 \\
(0,011)\end{array}$ & $\begin{array}{c}0,001 \\
(0,011)\end{array}$ & $\begin{array}{l}0,0018 \\
(0,011)\end{array}$ \\
\hline Phi (Precisión) & $\begin{array}{c}4,7028 * * * \\
(0,122)\end{array}$ & $\begin{array}{c}4,738^{* * * *} \\
(0,123)\end{array}$ & $\begin{array}{c}4,8471^{* * * *} \\
(0,126)\end{array}$ & $\begin{array}{c}3,8008^{* * * * *} \\
(0,097)\end{array}$ & $\begin{array}{c}3,9047 * * * * \\
(0,100)\end{array}$ & $\begin{array}{c}4,8175 * * * \\
(0,125)\end{array}$ & $\begin{array}{c}5,0292 * * * \\
(0,131)\end{array}$ & $\begin{array}{c}5,2746^{* * * *} \\
(0,138)\end{array}$ & $\begin{array}{c}4,9191 * * * * \\
(0,128)\end{array}$ & $\begin{array}{c}5,1564 * * * \\
(0,135)\end{array}$ & $\begin{array}{c}3,4088^{* * * *} \\
(0,085)\end{array}$ & $\begin{array}{c}3,875^{* * *} \\
(0,097)\end{array}$ & $\begin{array}{c}3,8049 * * * \% \\
(0,095)\end{array}$ & $\begin{array}{c}3,6462^{* * * *} \\
(0,092)\end{array}$ & $\begin{array}{c}3,6496 * \% * \% \\
(0,092)\end{array}$ \\
\hline & 1329,832 & 1316,382 & 1362,825 & 1105,984 & 1141,971 & 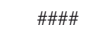 & 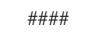 & 1432,353 & 1487,347 & 655,328 & 776,107 & 795,306 & 800,742 & 634,122 & 700,844 \\
\hline $\mathrm{N}$ & 2718 & 2718 & 2718 & 2718 & 2718 & 2718 & 2718 & 2718 & 2718 & 2718 & 2718 & 2718 & 2718 & 2718 & 2718 \\
\hline
\end{tabular}

uente: Elaboración propia. 
TABLA III

«Espera recibir Bienes, Dinero u otros incentivos materiales»

Y PROXIMIDAD A LAS REDES PARTIDARIAS EN ARGENTINA

\begin{tabular}{cccccc}
\hline & PJ & UCR & ARI & PRO & PPP \\
\hline & $-1,16$ & $-0,932$ & $-1,043$ & $-1,345$ & $-1,105$
\end{tabular}
«Espera recibir un Trabajo en el Sector Público» «Espera recibir la Obra Pública que requiere la Comunidad»

$\begin{array}{llllllllll}-0,406 & -0,694 & -1,124 & -1,362^{*} & -1,008 & -0,509 & -0,975 & -0,929 & -0,657 & -0,407\end{array}$

$(1,012) \quad(0,880) \quad(0,782) \quad(0,802)-(0,792)$
$\begin{array}{lllll}-0,004 & -0,005 & 0,008 & -0,017^{* * * *} & -0,005\end{array}$

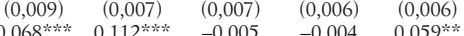

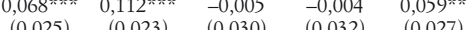

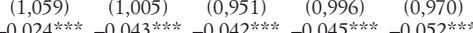
$\begin{array}{llll}(1,092) & (0,950) & (0,889) \quad(0,937) & (0,940)\end{array}$ $(0,009) \quad(0,007) \quad(0,007)-(0,007)-(0,007)$

$\begin{array}{llllll}\text { Ideología } & (0,009) & (0,007) & (0,007) & (0,007) & (0,007)\end{array}$ $\begin{array}{lllll}(0,026) & (0,025) & (0,033) & (0,038) & (0,031)\end{array}$ $\begin{array}{ccccc}(0,025) & (0,023) & (0,030) & (0,032) & (0,027) \\ 0,094^{* * * *} & 0,065^{* * * *} & 0,021 & 0,084^{* * * *} & 0,034\end{array}$ $(0,009)-(0,008)-(0,008)-0,045)-(0,007) \quad(0,007)$ $0,046 * \quad 0,122 * \% * * 0,007 \quad 0,174 * \% * 0,093 * \%$ $(0,026)-(0,026)-(0,035)-(0,040)-(0,032)$ \begin{tabular}{lccccc}
\multirow{2}{*}{ Red de activistas } & $0,07^{* * * *}$ & $0,049^{* * *}$ & $-0,024$ & 0,036 & 0,026 \\
& $(0,022)$ & $(0,020)$ & $(0,027)$ & $(0,030)$ & $(0,026)$
\end{tabular}

Edad $\begin{array}{ccccc}0,426 & 0,403 & 0,264 & 0,468 & 0,498\end{array}$ $\begin{array}{lllllllll}0,058 & 0,153 & 0,095 & 0,243 & 0,096 & 0,102 & 0,641 & 0,269 & 0,135\end{array}$

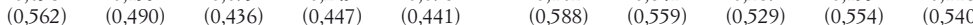
$\begin{array}{llllllllll}-0,06 & -0,055 & -0,038 & -0,058 & -0,041 & -0,034 & -0,107 & -0,046 & -0,027 & -0,036\end{array}$

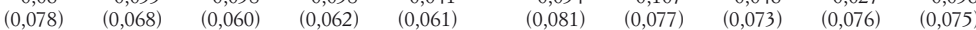
$\begin{array}{lllllllll}0,025 & 0,021 & 0,016 & 0,004 & 0,007 & 0,011 & 0,033 & -0,025 & -0,028\end{array}$

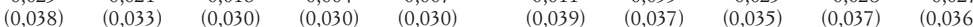

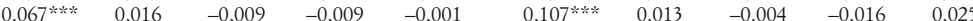

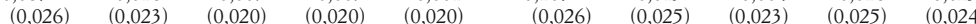

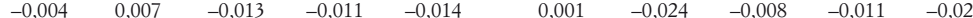

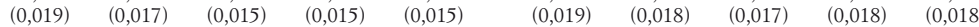

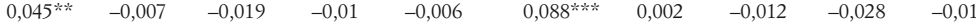

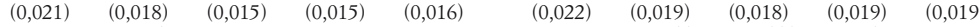

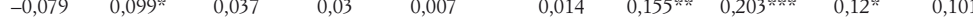

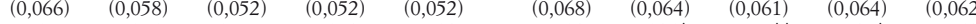
$\begin{array}{llllllllll}-0,055 & 0,014 & 0,01 & 0,018 & 0,009 & 0,008 & 0,084 * & 0,103 \cdots \cdots & 0,091 * & 0,049\end{array}$

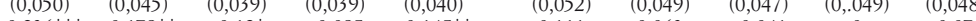

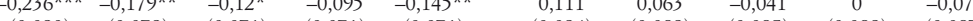

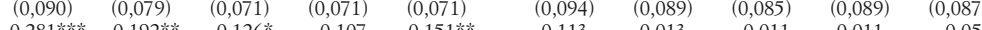
$\begin{array}{lllllllll}-0,281 * 0,192 & -0,126 & -0,107 & -0,151 * * & 0,113 & 0,013 & -0,011 & 0,011 & -0,05\end{array}$

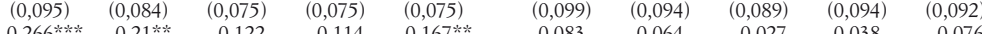

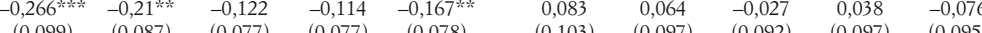

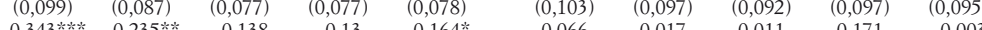

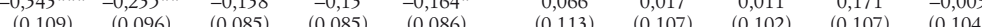

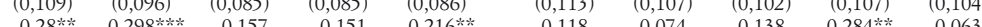

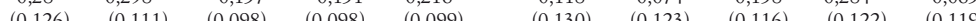

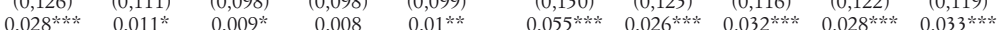
$(0,006)-(0,006)-(0,005)-(0,005)-(0,005)-(0,0,007)-(0,006)-(0,006)-(0,006)-(0,006)$

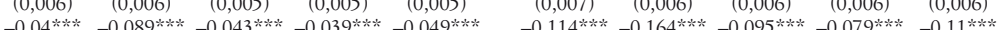

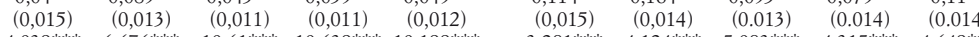

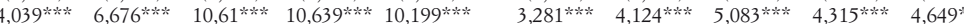
$\begin{array}{llllllllll}(0,105) & (0,180) & (0,289) & (0,290) & (0,278) & (0,081) & (0,105) & (0,132) & (0,112) & (0,121)\end{array}$

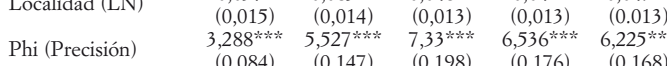
\begin{tabular}{llllll}
\hline LogLik & 641,765 & 1042,894 & 1294,637 & 1230,199 & 1188,455
\end{tabular} $\begin{array}{ccccc}790,610 & 1154.982 & 1611,684 & 1643,009 & 1604,47 \\ 2663 & 2663 & 2663 & 2663 & 2663\end{array}$ $\begin{array}{ccccc}247,631 & 492,715 & 707,125 & 634,122 & 700,84 \\ 2663 & 2663 & 2663 & 2663 & 2663\end{array}$

ज $\quad$ Nota: Modelo de regresión Beta con rango de variable dependiente de 0 (improbable) a 1 (extremadamente probable). 
Los resultados estadísticos proporcionan una gran cantidad de información apoyando, en términos generales, las hipótesis detalladas anteriormente. Una comparación visual del efecto de la distancia ideológica y de la proximidad de la red sobre las expectativas de recibir bienes (Gráfico II) muestra que la proximidad de los militantes partidarios del PJ es un predictor con significación estadística de las expectativas de los encuestados de recibir dádivas, empleo público y fondos públicos para obra pública por parte del mismo partido. De manera similar, la proximidad a la red de activistas de la UCR aumenta las expectativas de recibir dádivas, empleos y obra pública entre los votantes de la UCR. Las figuras no muestran evidencias que sostengan que la proximidad de los activistas a los pequeños partidos en Argentina aumente las expectativas de recibir bienes.

El Gráfico II también muestra que la proximidad de los activistas de un partido no tiene efectos sobre las expectativas distributivas de recibir dádivas entre los votantes chilenos mientras que la distancia ideológica se mantiene como un fuerte predictor para los tres partidos de la Concertación. Las diferencias entre los partidos grandes y pequeños en Argentina y entre los encuestados de Argentina y Chile son más llamativas con respecto a la distribución de dádivas y de empleos públicos. En cambio, en ambos países los resultados muestran que la distancia ideológica y la proximidad son importantes determinantes sobre las expectativas de recibir las obras públicas que la comunidad necesita (aunque la magnitud del efecto sea más débil para el PJ en el caso argentino).

Como se puede ver en la Tabla II, la distancia ideológica es significativa estadísticamente (y en la dirección negativa esperada) cuando intenta explicar la expectativa de los votantes de recibir beneficios por parte de los partidos de la Concertación en Chile. A mayor distancia ideológica de los votantes, menor es la expectativa de recibir beneficios. Los efectos de la distancia ideológica son significativos estadísticamente para todos los partidos de la Concertación, dando lugar a una disminución del $\approx 3,5 \%$ en la expectativa de recibir beneficios del PS y de la DC para cada unidad de aumento en la distancia ideológica medida en una escala del uno al diez. El efecto es más moderado para el PPD $(\approx 2)$ y estadísticamente insignificante para los partidos de derecha, UDI y RN, consistentes con un discurso político que no pone énfasis en la distribución sino que defiende el libre mercado. La proximidad de los militantes partidarios, contrariamente, no tiene efecto estadísticamente significativo en las expectativas distributivas de recibir beneficios por parte de ninguno de los cinco partidos políticos en Chile. En contra de lo esperado, los niveles socioeconómicos y educativos no tienen significancia estadística entre los votantes chilenos. Este resultado es consistente, sin embargo, con un trabajo cualitativo que muestra que los partidos chilenos distribuyen distintos tipos de bienes y servicios atendiendo a poblaciones con diferentes niveles socioeconómicos ${ }^{18}$. Por el contrario, tal como se esperaba, ni la proximidad de los beneficiarios del programa Chile Solidario ni la proximidad a los individuos que participan en las primarias partidarias

18. Mientras que los productos distribuidos van desde alimentos y medicinas a gafas de hipermetropía para los votantes más viejos, los servicios incluyen bienes repartidos por médicos, veterinarios, payasos, peluqueros e incluso los lectores del tarot. 
ERNESTO CALVO Y MARÍA VICTORIA MURILLO

CUANDO LOS PARTIDOS POLÍTICOS SE ENCUENTRAN CON SUS VOTANTES: UN ANÁLISIS DE LOS VÍNCULOS POLÍTICOS A TRAVÉS DE LAS REDES PARTIDARIAS Y LAS EXPECTATIVAS DISTRIBUTIVAS EN ARGENTINA Y CHILE

\section{GRÁFICO II}

EFECTO MARGINAL DE LA DISTANCIA IDEOLÓGICA Y DE LAS REDES PARTIDARIAS EN LAS EXPECTATIVAS DE RECIBIR DÁDIVAS, EMPLEOS EN EL SECTOR PÚBLICO U OBRAS PÚBLICAS PARA LA COMUNIDAD

Distancia Ideológica, Argentina

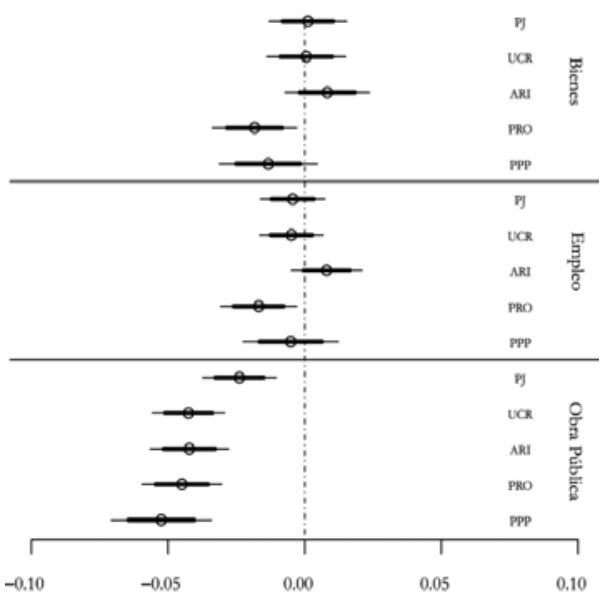

Efecto de Distancia Ideológica en Expectativas Distributivas
Distancia Ideológica, Chile

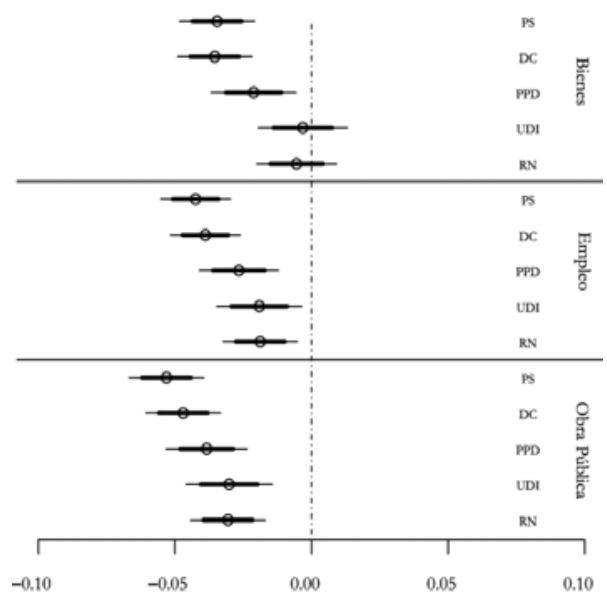

Efecto de Distancia Ideólogica en Expectativas Distributivas

Proximidad a la Red de Activistas, Argentina Proximidad a la Red de Activistas, Chile

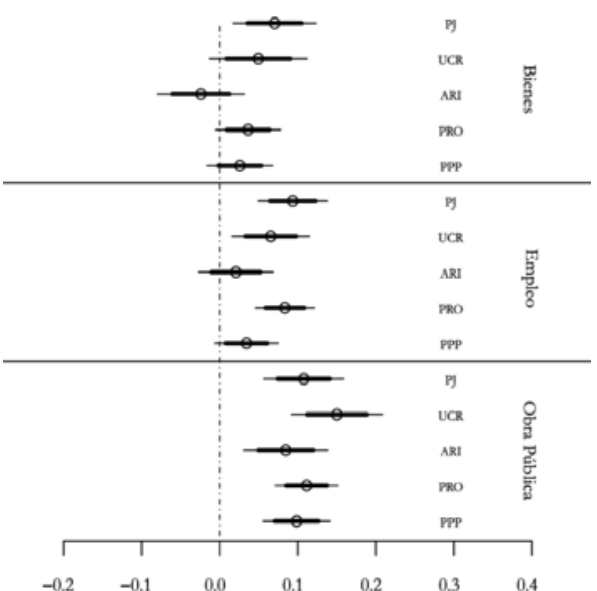

Efecto de la Red de Activistas en las Expectativas Distributivas

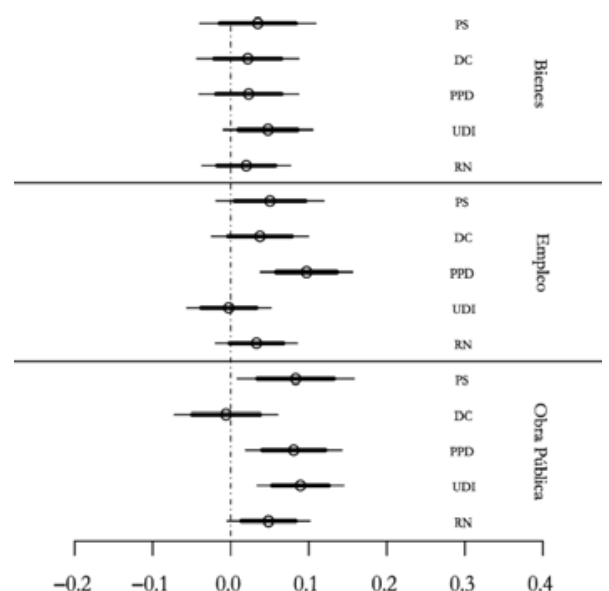

Efecto de la Red de Activistas en las Expectativas Distributivas

Nota: Los gráficos describen el coeficiente estimado en las Tablas II y III, con intervalos de confianza de 0,05 (línea delgada) y al nivel ,1 (línea gruesa). 
aumenta la probabilidad percibida por parte de los votantes chilenos de recibir beneficios. Finalmente, la visión de los encuestados sobre la redistribución tiene efecto positivo. Y dado que la densidad de población genera un efecto positivo en todos los partidos, esto permite suponer que la visión positiva de la redistribución ocurre en todas las grandes ciudades.

La Tabla III muestra que en Argentina, contrariamente, la distancia ideológica no tiene efecto significativo en las expectativas de los encuestados de recibir dádivas del PJ o de la UCR, mientras que la proximidad a los militantes partidarios tiene un fuerte efecto positivo tanto para el PJ como para la UCR. Ni la ideología ni la proximidad a los activistas explica las expectativas distributivas de los votantes del ARI. Mientras que la ideología de los militantes es un predictor significativo de las expectativas distributivas del PRO. Como era de esperar, también se encontró un efecto significativo de la proximidad de los beneficiarios de los programas Jefes y Jefas al PJ. Otras variables de control tienen los efectos esperados. Las altas expectativas de recibir beneficios están asociadas a una visión positiva sobre la distribución de dádivas. Y la proximidad a las personas que participan en las primarias del partido aumenta las expectativas distributivas de recibir dádivas del PJ entre los encuestados. A diferencia de Chile, se encuentra que la densidad de población tiene un efecto negativo sugiriendo el comportamiento de los votantes en las pequeñas localidades, tal como lo afirmaron Brusco et al. (2004). Finalmente, en Argentina un nivel socioeconómico alto tiene un resultado negativo y un efecto estadístico significativo en los beneficios esperados percibidos por todos los partidos (con la categoría de los votantes más pobres como línea de base).

Las expectativas distributivas con respecto a empleos del sector público muestran patrones similares a los de las dádivas. La probabilidad percibida de recibir el ofrecimiento de un puesto de trabajo en el sector público disminuye con la distancia ideológica para los cinco partidos y aumenta con el incremento en el nivel socioeconómico del PPD y la UDI -el efecto es positivo, pero no significativo para la DC, PS y RN-. La proximidad a los activistas del partido tiene un efecto estadísticamente significativo sólo en los casos del PS y el PPD.

En Argentina, la probabilidad percibida de recibir el ofrecimiento de un puesto de trabajo en el sector público aumenta en la medida en que los encuestados están más conectados con las redes tanto de activistas como de candidatos del PJ y la UCR. El efecto también es más fuerte para el caso de los empleos públicos que para las dádivas. Estos resultados sugieren que los empleos son mejores que las dádivas para llegar hasta los principales simpatizantes que se desempeñan como agentes para conseguir votos para el partido. En el caso del PJ, conocer un mayor número de activistas (una desviación estándar por encima de la tasa de prevalencia) aumenta la expectativa de recibir el ofrecimiento de un empleo público en un $8,65 \%$. El efecto es más moderado para la UCR, llevando a un aumento del $5,26 \%$ si el encuestado conoce un mayor número de activistas (una desviación estándar por encima de la tasa de prevalencia). Las variables de control tienen los efectos esperados. Al igual que con las dádivas, la proximidad con la red de las primarias del PJ tiene un efecto positivo y significativo. La densidad de población tiene un efecto negativo significativo tanto para el PJ y la UCR, en consonancia con la 
literatura que muestra la dependencia de la población de las provincias menos urbanizadas respecto al empleo público en Argentina (Gibson y Calvo 2000).

Por último, se analizan las expectativas acerca de la entrega de fondos para hacer obra pública, si un partido gana en el distrito del entrevistado. Las expectativas fueron menos claras en este caso debido a la naturaleza no exclusiva de las obras públicas a nivel local, y se encuentra que los votantes en ambos países identifican tanto a las redes como a la ideología como mecanismos para la distribución de bienes públicos. En Chile y Argentina, la distancia ideológica y la proximidad con los miembros del partido tuvieron los efectos esperados -aunque los efectos de las redes no son significativos para los miembros de la DC y de la RN-. En Chile, el efecto del nivel socioeconómico es positivo aun cuando su significación varía. En concordancia con lo antedicho, el impacto del programa asistencial Chile Solidario es negativo y significativo para los tres partidos de la Concertación. En Argentina, es importante notar el declive en la relevancia de las variables socioeconómicas y el efecto positivo de la educación, excepto para el caso del PJ. Esto es, el estatus socioeconómico parece no tener efecto en la expectativa de los votantes de recibir obra pública para sus comunidades. Sin embargo, los votantes con menores niveles educativos con vínculos tradicionales con los peronistas perciben al PJ como un partido que tiende a repartir bienes para sus distritos. Finalmente, la red de militantes que participan en las primarias tiene un efecto positivo para los partidos de la Concertación en Chile y para el PJ en Argentina. Esta asociación de la obra pública con la participación política en las primarias es consistente con la literatura en Argentina que muestra que la participación en las primarias peronistas (y los actos públicos) puede ser fácilmente monitoreada por los intendentes y retribuida mediante la asignación de obra pública, especialmente durante el período electoral. Sin embargo, este efecto no había sido identificado previamente para Chile, a pesar de que afecta a los tres partidos en el momento de la encuesta.

En suma, los votantes chilenos forman sus expectativas de acuerdo a características ideológicas en el caso de todos los tipos de bienes, aun para el caso de las dádivas para los partidos de la Concertación. Mientras tanto los votantes de Argentina confían más en la proximidad a los militantes del PJ y de la UCR cuando forman sus expectativas distributivas en el caso de las dádivas y de los empleos públicos y el efecto disminuye para el caso de la obra pública, que es el tipo de beneficio menos exclusivo de los tres tipos de bienes recibidos a nivel local. La distancia ideológica moldea las expectativas distributivas del PRO para los tres tipos de beneficios. Esto es, en Argentina, el impacto de las redes políticas es diferente para los dos partidos políticos más estables debido a una mayor extensión de su capacidad organizativa y a las dificultades de ubicar ideológicamente estos partidos. Estos resultados sugieren que aun cuando en ambos países los encuestados confían en la extensión de las redes políticas, estas redes juegan un rol sumamente importante a la hora de definir las expectativas distributivas de los votantes del PJ y la UCR en Argentina. Los hallazgos presentes en este trabajo confirman las predicciones a nivel nacional con respecto a los diferentes vínculos entre los partidos políticos y los electores (programáticos o clientelares), a pesar de los recientes debates entre los investigadores chilenos con respecto a la creciente influencia del clientelismo. 
Aunque se encuentran evidencias de la existencia de clientelismo en Chile, en consonancia con trabajos recientes (Luna 2010), los resultados de esta investigación muestran que los votantes chilenos siguen confiando en los aspectos ideológicos aun a la hora de definir sus expectativas con relación a recibir beneficios. El hecho de que no se encuentra el efecto mencionado para el caso de la UDI en relación con los bienes repartidos confirma el argumento de Luna (2010) sobre el hecho de que los electores de este partido financian la distribución de beneficios a votantes distantes ideológicamente. Finalmente, los resultados muestran que tanto las redes partidarias como la ideología influyen en las expectativas distributivas de las obras públicas en ambos países debido al carácter menos exclusivo del bien otorgado en el nivel local.

\section{CONCLUSIÓN}

En este artículo se propone una nueva conceptualización de los vínculos políticos programáticos o clientelares basados en las expectativas distributivas de los votantes. Desde el punto de vista de los votantes, se afirma que los partidos programáticos y clientelares difieren en la forma en que distintos tipos de beneficios son distribuidos. En el primer caso, los votantes esperan que las dádivas, el empleo público y la obra pública sea repartida por las redes de militantes. En el segundo caso, los votantes anticipan que los bienes serán distribuidos mediante una política pública cuando los partidos con proximidad ideológica tienen acceso a los recursos públicos. Para probar el modelo, se propone una nueva metodología para medir los vínculos entre votantes y partidos políticos tanto en términos ideológicos como organizativos. Esta contribución al estudio comparado de los partidos políticos es luego puesta a prueba en dos países, mostrando la variación entre y dentro los sistemas de partidos.

Este estudio realiza dos contribuciones principales. En primer lugar, se enfatiza el valor de poner el foco en el acceso a beneficios mediante fondos públicos para analizar los vínculos entre el partido y el votante visto desde la perspectiva del votante y poder predecir patrones de variaciones de dichos vínculos. En particular, el estudio explora cómo la capacidad organizativa y la identificación ideológica (entre partidos) y los límites institucionales sobre la discreción de las políticas públicas (entre países) moldean estos vínculos. Segundo, propone una nueva metodología comparativa para medir los vínculos entre votantes y partidos que puede ser usada en otros casos, incluso cuando las redes de distribución no son partidarias.

Este artículo constituye una contribución importante para la evaluación de los vínculos entre los partidos políticos y los electores desde la perspectiva de los votantes, centrándose en el tipo de acceso a los bienes exclusivos. Los partidos políticos ofrecen un conjunto de bienes exclusivos y no exclusivos para los votantes. Sin embargo, los mecanismos que determinan el acceso a los bienes, ya sea a través de algunos criterios de política general o a través de las redes políticas, moldean las percepciones de los votantes con respecto a la forma en que se distribuirán los beneficios en el futuro. Los votantes tienen que definir sus propias expectativas de distribución con respecto a los dos tipos de bienes con el fin de actuar sobre esas expectativas a la hora de votar. 
ERNESTO CALVO Y MARÍA VICTORIA MURILLO

CUANDO LOS PARTIDOS POLÍTICOS SE ENCUENTRAN CON SUS VOTANTES: UN ANÁLISIS DE LOS VÍNCULOS POLÍTICOS A TRAVÉS DE LAS REDES PARTIDARIAS Y LAS EXPECTATIVAS DISTRIBUTIVAS EN ARGENTINA Y CHILE

Es importante destacar que es la experiencia en el modo de acceso a los beneficios exclusivos lo que moldea las expectativas de los votantes al generar previsibilidad con respecto a los patrones de distribución. Sin embargo, las diferencias en el acceso a las prestaciones no sólo son definidas en función de las restricciones institucionales que generan variaciones entre los sistemas políticos, sino también por las diferencias en la capacidad de organización y la dotación de los recursos fiscales dentro de los sistemas políticos. Por lo tanto, en la clasificación de los vínculos entre los partidos políticos y los votantes es importante tener en cuenta que los partidos ofrecen diferentes tipos de bienes y que los votantes valorarán tanto los bienes exclusivos como los no exclusivos en función de sus propias experiencias y del beneficio marginal que les representa.

Por otra parte, este artículo presenta una novedosa metodología que permite comparaciones transversales de los vínculos entre los partidos políticos y los electores sobre la base de patrones de acceso a los beneficios financiados con fondos públicos. Esta metodología nos permite medir el tamaño y la estructura de las redes partidarias, así como estimar el efecto de las redes y de la proximidad ideológica sobre las expectativas distributivas de los votantes. Las estimaciones que se derivan proporcionan una herramienta comparativa fundamental en la medición de la naturaleza de los vínculos entre los partidos políticos y los votantes a través de diferentes contextos. Esta metodología se puede aplicar en otros casos para descubrir los vínculos políticos entre los partidos y los votantes. Por otra parte, también se puede aplicar a redes no partidarias. Por ejemplo, en contextos en los que el origen de las redes de distribución de los beneficios públicos no proviene de un partido político, sino de una organización religiosa o étnica, se espera que esta metodología pueda evaluar el impacto de este tipo de redes en las expectativas distributivas de los votantes. En efecto, esta metodología podría ser utilizada para probar si el impacto electoral de tales categorías adscriptivas se basa en la identificación descriptiva o en el acceso a los beneficios excluibles, que es un debate fundamental en la literatura existente sobre el impacto de las identidades étnicas en la política.

Para concluir, este artículo ofrece una nueva forma de entender la vinculación entre los partidos y los votantes al mostrar que los partidos generan diferentes expectativas distributivas para los votantes, quienes poseen distinta sensibilidad de acuerdo a la proximidad con los miembros del partido. Por ello se considera que sería fructífero que futuros trabajos sobre políticas distributivas empeñaran sus esfuerzos en explorar no solamente las diferencias en las demandas de distribución de los votantes en función del impacto de los diferentes tipos de acceso a las prestaciones, sino también en el efecto que tiene el acceso a los beneficios sobre los determinantes de la decisión de voto individual. Los futuros trabajos deberían, entonces, centrarse en las estrategias electorales de los partidos políticos de acuerdo a diferentes expectativas de los votantes, a las limitaciones institucionales y a diversas dotaciones de la capacidad de organización y de los recursos fiscales. 
ERNESTO CALVO Y MARÍA VICTORIA MURILLO CUANDO LOS PARTIDOS POLIITICOS SE ENCUENTRAN CON SUS VOTANTES: UN ANÁLISIS DE LOS VÍNCULOS POLÍTICOS A TRAVÉS DE LAS REDES PARTIDARIAS Y LAS EXPECTATIVAS DISTRIBUTIVAS EN ARGENTINA Y CHILE

VIII. BIBLIOGRAFÍA

Banfield, Edward C. y Wilson, James Q. City politics. Cambridge: Harvard University Press, 1963.

BAU AEDO, Catalina. Experiencias exitosas en la profesionalización de la función pública en América Latina. El caso Chile. En Foro Iberoamericano: Revitalización de la administración pública. Estrategias para la Implantación de la Carta Iberoamericana de la Función Pública. México, 2005.

Brusco, Valeria; NAZAReno, Marcelo y Stokes, Susan C. Vote Buying in Argentina. Latin American Research Review, 2004, vol. 39 (2): 66-88. http://dx.doi.org/10.1353/lar.2004.0022

Cox, Gary W. Swing Voters, Core Voters and Distributive Politics. En SHAPIRO, Ian; STOKES, Susan; WoOD, Elizabeth y KIRSHNER, Alexander (eds.). Political Representation. New York: Cambridge University Press, 2009: 342-357.

Cox, Gary W. y McCubBIns, Mathew D. Electoral Politics as a Redistributive Game. The Journal of Politics, 1986, vol. 48 (2): 370-389. http://dx.doi.org/10.2307/2131098

DíAZ-CAYEROS, Alberto. Electoral risk and redistributive politics in Mexico and the United States. Studies in Comparative International Development, 2008, vol. 43 (2): 129-150. http://dx.doi.org/10.1007/s12116-008-9020-1

DixiT, Avinash y LONDREGAn, John. The Determinants of Success of Special Interests in Redistributive Politics. The Journal of Politics, 1996, vol. 58 (4): 1132-1155. http://dx.doi.org/10.2307/2960152

GELMAN, Andrew y HILL, Jennifer. Data analysis using regression and multilevel/hierarchical models, Analytical methods for social research. New York: Cambridge University Press, 2007.

GIRAUDI, Agustina. The Distributive Politics of Emergency Employment Programs in Argentina (1993-2002). Latin American Research Review, 2007, vol. 42 (2): 33-55. http://dx.doi.org/10.1353/lar.2007.0021

Huneeus, Carlos. The Pinochet regime. Boulder: Lynne Rienner Publishers, 2007.

KeEFER, Philip y Vlaicu, Razvan. Democracy, credibility, and clientelism. Journal of Law Economics E Organization, 2008, vol. 24 (2): 371-406.

KemaHLIOGLU, Ozge. When the agent becomes the boss: the politics of public employment in Argentina and Turkey. Phd Thesis, Columbia University, 2006.

KirchHeIMER, Otto. The Transformation of the Western European Party System. En LA PALOMBARA, Joseph y WeIneR, Myron (eds.). Political Parties and Political Development. Princeton: Princeton University Press, 1966: 177-200.

KitsCHELT, Herbert. Linkages between citizens and politicians in democratic politics. Comparative Political Studies, 2000, vol. 33 (6-7): 845-879. http://dx.doi.org/10.1177/001041400003300607

Kitschelt, Herbert; Hawkins, Kirk A.; LunA, Juan Pablo; Rosas, Guillermo y ZeChmeister, Elizabeth J. Latin American Party Systems, Cambridge studies in comparative politics. New York: Cambridge University Press, 2010.

Kitschelt, Herbert y WiLkinson, Steven. Patrons, clients, and policies: patterns of democratic accountability and political competition. New York: Cambridge University Press, 2007.

LIPSET, Seymour Martin y ROKKAN, Stein. Party systems and voter alignments: cross-national perspectives. Contributors: Robert R. Alford and others, International yearbook of political bebavior research v. 7. New York: Free Press, 1967. 
ERNESTO CALVO Y MARÍA VICTORIA MURILLO

CUANDO LOS PARTIDOS POLÍTICOS SE ENCUENTRAN CON SUS VOTANTES: UN ANÁLISIS DE LOS VÍNCULOS

POLÍTICOS A TRAVÉS DE LAS REDES PARTIDARIAS Y LAS EXPECTATIVAS DISTRIBUTIVAS EN ARGENTINA Y CHILE

LunA, Juan Pablo. Segmented Party Voter Linkages in Latin America: The Case of the UDI. Journal of Latin American Studies, 2010, vol. 42 (2): 325-356.

http://dx.doi.org/10.1017/S0022216X10000465

LunA, Juan Pablo y MARDONES, Rodrigo. Distributive Politics in a Non-Machine Party System: The Allocation of Targeted Social Funds and Subsidies in Chile (2000-2008). Santiago: Universidad Católica de Chile, 2009.

Magaloni, Beatriz Magaloni; Díaz-Cayeros, Alberto y Estévez, Federico. Clientelism and portfolio diversification: a model of electoral investment with applications to Mexico. En KITSCHELT, Herbert y WILKINSON, Steven (eds.). Patrons, clients, and policies: patterns of democratic accountability and political competition. New York: Cambridge University Press, 2007: 182-205.

McCarty, Christopher; KILlworth, Peter D.; Bernard, H. Russell; JohnSen, Eugene y Shelley, Gene A. Comparing two methods for estimating network size. Human Organization, 2000, vol. 60 (1): 28-39.

Nichter, Simeon. Vote Buying or Turnout Buying? Machine Politics and the Secret Ballot. American Political Science Review, 2008, vol. 102 (1): 19-31.

PANEBIANCO, Angelo. Political parties: organization and power, Cambridge studies in modern political economies. New York: Cambridge University Press, 1988.

REHREN, Alfredo J. Clientelismo político y reforma del Estado en Chile. Santiago: Centro de Estudios Públicos, 2000.

SCHAFFER, Frederic C. Elections for Sale: The Causes and Consequences of Vote Buying. Boulder: Lynne Rienner Publishers, 2007.

SCHMIDT, Steffen W. Friends, followers and factions: a reader in political clientelism. Berkeley: University of California Press, 1977.

Scotт, James C. Patron-Client Politics and Political Change in Southeast Asia. American Political Science Review, 1972, vol. 66 (1): 91-113. http://dx.doi.org/10.2307/1959280

STOKES, Susan. C. Perverse accountability: A formal model of machine politics with evidence from Argentina. American Political Science Review, 2005, vol. 99 (3): 315-325.

StOKes, Susan C. Political Clientelism. En Goodin, Robert E. Oxford Handbooks of political science. New York: Oxford University Press, 2007: 648-672.

SzWARCBERG, Mariela. Counting Heads and Votes: Authoritarian and Democratic Strategies of Electoral Mobilization in Argentina. Paper presentado en Annual meeting of the MPSA Annual National Conference. Palmer House Hotel, Hilton, Chicago, IL, 2008.

SzWARCBERG, Mariela. Uncertainty, Political Clientelism, and Voter Turnout in Latin America: Why Parties Conduct Rallies in Argentina. Comparative Politics, 2012, vol. 45 (1): 88-106. http://dx.doi.org/10.5129/001041512802822851

UjhelyI, Gergely y CALvo, Ernesto. Political Screening: Theory and Evidence from the Argentine Public Sector. Working Paper. University of Houston.

Weitz-Shapiro, Rebecca. Partisanship and Protest: The Politics of Workfare Distribution in Argentina. Latin American Research Review, 2006, vol. 41 (3): 122-147. http://dx.doi.org/10.1353/lar.2006.0051

WEITZ-SHAPIRO, Rebecca. Choosing clientelism: political competition, poverty and social welfare policy in Argentina. PhD Dissertation. New York City, 2008.

Wellhofer, Spencer. The Political Incorporation of the Newly Enfranchised Voter - Organizational Encapsulation and Socialist-Labor-Party Development. Western Political Quarterly, 1981, vol. 34 (3): 399-414.

http://dx.doi.org/10.2307/447219 
ERNESTO CALVO Y MARÍA VICTORIA MURILLO

CUANDO LOS PARTIDOS POLÍTICOS SE ENCUENTRAN CON SUS VOTANTES: UN ANÁLISIS DE LOS VÍNCULOS POLÍTICOS A TRAVÉS DE LAS REDES PARTIDARIAS Y LAS EXPECTATIVAS DISTRIBUTIVAS EN ARGENTINA Y CHILE

Zheng, Tian; Salganik, Matthew y Gelman, Andrew. How many people do you know in Prison?: using overdispersion in count data to estimate social structure in networks. Journal of the American Statistical Association, 2006, vol. 101 (474): 409-423.

http://dx.doi.org/10.1198/016214505000001168 